Article

\title{
Measured Performance of a Mixed-Use Commercial-Building Ground Source Heat Pump System in Sweden
}

\author{
Jeffrey D. Spitler ${ }^{1, *}$ and Signhild Gehlin ${ }^{2}$ \\ 1 School of Mechanical and Aerospace Engineering, Oklahoma State University, Stillwater, OK 74078, USA \\ 2 Swedish Geoenergy Center, Västergatan 11, 22104 Lund, Sweden; signhild@geoenergicentrum.se \\ * Correspondence: spitler@okstate.edu
}

Received: 3 May 2019; Accepted: 20 May 2019; Published: 27 May 2019

\begin{abstract}
When the new student center at Stockholm University in Sweden was completed in the fall of 2013 it was thoroughly instrumented. The $6300 \mathrm{~m}^{2}$ four-story building with offices, a restaurant, study lounges, and meeting rooms was designed to be energy efficient with a planned total energy use of $25 \mathrm{kWh} / \mathrm{m}^{2} /$ year. Space heating and hot water are provided by a ground source heat pump (GSHP) system consisting of five $40 \mathrm{~kW}$ off-the-shelf water-to-water heat pumps connected to 20 boreholes in hard rock, drilled to a depth of $200 \mathrm{~m}$. Space cooling is provided by direct cooling from the boreholes. This paper uses measured performance data from Studenthuset to calculate the actual thermal performance of the GSHP system during one of its early years of operation. Monthly system coefficients-of-performance and coefficients-of-performance for both heating and cooling operation are presented. In the first months of operation, several problems were corrected, leading to improved performance. This paper provides long-term measured system performance data from a recently installed GSHP system, shows how the various system components affect the performance, presents an uncertainty analysis, and describes how some unanticipated consequences of the design may be ameliorated. Seasonal performance factors (SPF) are evaluated based on the SEPEMO ("SEasonal PErformance factor and MOnitoring for heat pump systems") boundary schema. For heating (" $\left.\mathrm{H}^{\prime \prime}\right)$, SPFs of $3.7 \pm 0.2$ and $2.7 \pm 0.13$ were obtained for boundaries $\mathrm{H} 2$ and $\mathrm{H} 3$, respectively. For cooling ("C"), a C2 SPF of $27 \pm 5$ was obtained. Results are compared to measured performance data from 55 GSHP systems serving commercial buildings that are reported in the literature.
\end{abstract}

Keywords: ground source heat pump; ground heat exchanger; borehole thermal energy storage; office building; system performance; uncertainty analysis

\section{Introduction}

Buildings in developed countries consume $20 \%-40 \%$ of the total final energy consumption [1]; in 2017, residential and commercial buildings consumed 39\% [2] of all energy in the USA. Of this, about half [1] is used to provide heating, ventilation, and cooling (HVAC). Likewise, in Sweden, in 2016, buildings consumed 39\% of all energy [3] and 55\% of that quantity was used for space heating and domestic hot water (DHW) [4]. An extensive amount of research, development, regulation, and investment has gone towards reducing energy consumption of building HVAC systems. Nevertheless, actual building energy consumption is often significantly higher than that planned at the design stage. This difference is sometimes referred to as the "performance gap" [5-8].

The performance gap is particularly of concern for green, low energy buildings. Scofield [9] examined site energy use intensities (Energy use intensities are a measure of building energy 
consumption, usually given as annual energy consumption per square meter or square foot of building floor area.) for 100 LEED-certified buildings. ("Leadership in Energy and Environmental Design" is a widely used green building rating system developed by the US Green Building Council.) He found no energy savings for the buildings as a group when compared to comparable buildings in the US commercial building stock. (Individual buildings did show savings, but when considered as a group, no statistically-significant savings were found.) Scofield [10] examined 953 buildings in New York City, of which 21 were LEED-certified, and found no savings as compared with non-LEED-certified buildings.

Li et al. [11] investigated 51 office buildings certified as "high-performance" in the US, Europe, and Asia. Surprisingly, almost half of the buildings did not even meet the American Society of Heating, Refrigerating and Air-conditioning Engineers (ASHRAE) Standard 90.1-2004 energy targets, and energy use intensities among the buildings varied by a factor of 11 . They concluded that no single factor determined the actual energy performance of these buildings and that adding multiple efficient technologies doesn't guarantee good energy performance.

Kurkinen et al. [12] compiled 21 case studies of Swedish buildings (mainly multi-family), built between 2002 and 2013. Five of the 21 buildings exhibited an energy performance gap of more than $20 \%$ compared to design calculations. The 21 case studies were complemented with a literature review of 20 studies from 1988-2014 on the building energy performance gap and its causes in Sweden and the Nordic countries. Most of these studies report on residential buildings and the reported performance gaps are as high as $250 \%$.

Geng et al. [13] performed a meta-analysis of the operating performance of green buildings, primarily located in the US and China. A literature review identified 106 studies with significant quantitative analysis of the data. Energy use intensities for certified high performance buildings were compared-121 buildings from the US and 31 buildings from China. Only a slight improvement in average building performance could be found as the certification level improved. Within each certification level, individual performance varied widely.

The above studies rely on measurements of energy use intensity (EUI). Energy use intensity is commonly used for such studies because it is relatively easy to measure, needing as a minimum only building utility bills and floor area, and hence EUIs are widely available. The wide availability has facilitated compilation of large databases such as the US Department of Energy (DOE) Buildings Performance Database [14], covering about 750,000 buildings, of which 44,000 are commercial and the remainder residential. Such a large data set allows a variety of "big data" approaches to analysis, including linear regression to estimate retrofit savings [15], machine learning [16,17], and clustering analysis [18] to predict energy consumption. In many cases, submetering also allows EUIs for lighting and plug loads to be determined, further enhancing the value of these analyses.

Buildings that utilize ground-source heat pump (GSHP) systems follow one of the general trends in EUI discussed above-specifically, the actual performance may vary widely. In a study of 35 commercial-building GSHP systems where the overall building energy usage was ranked on a normalized energy use intensity scale [19], the Electric Power Research Assistant (EPRI) [20] showed percentile rankings all the way from 1 to 100 . That is, the measured EUIs varied even more widely than the 51 office buildings examined by Li et al. [11].

Despite the many advantages and opportunities of using large data sets of energy use intensities, analyses of EUIs have inherent limitations. Specifically, it is difficult to differentiate between the effects of the building envelope loads and occupants and the performance of the HVAC system, e.g., if the building has high energy use, what is the cause? A poor building envelope? High internal heat gains or other occupant effects? Poor HVAC system performance?

This paper focuses on an approach that can help resolve this question for a specific building-field monitoring of the HVAC system performance-specifically for a ground-source heat pump system in a university building in Stockholm, Sweden. This is inherently more difficult and expensive, requiring additional sensors and data acquisition systems. Unfortunately, there are very limited comparison data available, so even after carefully measuring performance of a single building system, there are 
relatively few comparable measurements that have been reported in the literature. Nevertheless, the decreasing cost of sensors and data acquisition suggest that these analyses may be more widely available in the future. Thus, one contribution of this paper is providing a comparison benchmark for future studies.

One critical issue for representing field performance is the boundary schema-where should the boundaries be drawn when determining the system performance? Early work on defining boundary schema was done for residential systems, so we first review some of the work on field measurement of residential-building GSHP systems before covering the main focus of the review, measured-performance of commercial-building GSHP systems.

\subsection{Literature Review—Residential Building GSHP System Performance Measurement}

For residential buildings, field measurements of heat pump system performance have been reviewed by Gleeson and Lowe [21]. The reviewed studies were undertaken in Austria, Denmark, Germany, Sweden, Switzerland, and the UK. The studies, which covered both ground-source and air-source heat pump systems, utilized sufficient instrumentation to measure system performance on a seasonal basis. Gleeson and Lowe identified two different boundary schema; both have multiple boundary definitions that may be applied. The boundary definitions differ as to which components are included-e.g., source-side circulating pump, load-side circulating pump, fans, etc.-and as to where heat delivered is measured, e.g., to the buffer tank or from the buffer tank to the heat distribution system. The authors attempted to harmonize the field trial results so that broad comparisons could be made between different field trials. Performance of over 600 heat pump installations was examined. One notable finding is an inconsistency in results that cannot readily be explained by the quality of the equipment or the building load profiles. For 216 GSHP installations, the seasonal performance factor that accounts for circulation pumps on both sides of the heat pumps and integrated backup heating ranged from 1.4 to 5.1. Gleeson and Lowe surmise that the issue is the quality of the design and installation.

A particular focus of the Gleeson and Lowe [21] paper is harmonizing the field trial results computed with different boundary schema, each of which has different boundaries. The different boundaries are important for several reasons-these include comparison to conventional systems, benchmarking of GSHP system performance, identification of best practices, determining the causes of poor performance, and giving guidance as to how system performance may be improved. One such schema was defined by the EU project SEPEMO [22], giving heating and cooling seasonal performance factors (SPFs) for a range of boundaries, as shown in Table 1.

The SEPEMO boundary scheme was applied to 44 heat pump systems in six European countries (Sweden, Germany, Greece, the Netherlands, France, and Austria) with varying heat sources (air, ground) and heat distribution methods (panel radiators, floor heating). All but five of these heat pump systems were installed in residential buildings, mostly single-family houses. Nordman [22] reports $\mathrm{SPF}_{\mathrm{H} 3}$ (heating system except the heat distribution inside the building) for the 44 heat pump systems, ranging from 1.3-7.3. Eight of the systems have $\mathrm{SPF}_{\mathrm{H} 3}$ below 2.6 and 15 systems (all GSHP systems) show $\mathrm{SPF}_{\mathrm{H} 3}$ above 4. Nordman [22] notes that heat pump system performance depends not only on the heat pump, but also on the climate and quality of installation. The SEPEMO project guidelines work well for smaller residential GSHP systems, but do not fully address all of the features that may be found in GSHP systems serving larger and more complex GSHP systems, such as commercial, institutional, and multi-family buildings.

Note that the SEPEMO system boundaries for $\mathrm{SPF}_{\mathrm{H} 1}$ and $\mathrm{SPF}_{\mathrm{H} 2}$ correspond to $\mathrm{SPF}_{\mathrm{C} 1}$ and $\mathrm{SPF}_{\mathrm{C} 2}$, while the boundaries for $\mathrm{SPF}_{\mathrm{H} 3}$ do not correspond directly to those for $\mathrm{SPF}_{\mathrm{C} 3}$. $\mathrm{SPF}_{\mathrm{H} 3}$ includes auxiliary heating but not distribution pumps/fans, whereas $\mathrm{SPF}_{\mathrm{C} 3}$ includes distribution pumps/fans, but not supplementary cooling units. Hence if there is no auxiliary heating in the heating system, $\mathrm{SPF}_{\mathrm{H} 2}=$ $\mathrm{SPF}_{\mathrm{H} 3}$, while $\mathrm{SPF}_{\mathrm{C} 3}=\mathrm{SPF}_{\mathrm{C} 4}$ for systems without supplementary cooling. 
Miara et al. [23] use a similar boundary scheme to the SEPEMO scheme, for residential heating only (Table 1). System boundaries 0-2 correspond to the SEPEMO boundaries $1-3$, while the fourth system boundary includes circulation pumps between back-up heater and storage tanks, but excludes the storage tanks and distribution system beyond the tanks. The field study by Miara et al. included 56 GSHP pump systems for small residential buildings over a period of three years. The reported average seasonal performance factors for the $56 \mathrm{GSHP}$ systems were 4.19 for $\mathrm{SPF}_{0}, 3.93$ for $\mathrm{SPF}_{1}$, 3.88 for $\mathrm{SPF}_{2}$, and 3.75 for $\mathrm{SPF}_{3}$. The DHW share was $18 \%$ over the three-year period. $\mathrm{SPF}_{2}$ varied in the range of 3.75-3.9 over the three years when the measurements took place.

Another two system boundary schema suitable for residential GSHP heating systems are found in Koeningsdorff [24] (pages 276-277) and in the German guidelines VDI 4650 [25]. VDI 4650 (Table 1) defines four system boundary levels, where the first only includes the heat pump unit (corresponds to the SEPEMO boundary H1), the second only includes the ground source with a load-side circulation pump, the third corresponds to the SEPEMO boundary H3, and the fourth boundary includes both the source-side and load-side. The Koenigsdorff scheme consists of seven system boundaries, of which the first three follow the VDI 4650 scheme, and the seventh corresponds to the fourth boundary in the VDI scheme.

\subsection{Literature Review—Commercial Building GSHP System Performance Measurement}

Commercial buildings often have considerably more complex GSHP systems than residential buildings. Beyond energy use intensity measurements, several authors have made measurements of system coefficient of performance (COP) for a single day [26,27], several days [28], or several weeks $[29,30]$.

Several authors [31-35] have presented detailed performance measurements of building heating systems that incorporate GSHP and other renewable sources such as solar-thermal and solar-electric on site. The first paper focuses on other aspects of the system performance. Wemhoener et al. [32,33] report monthly and annual performance factors, with an annual value of 3.92 for combined space heating and domestic hot water heating, accounting for the heat pump and source-side circulating pump. Lazzarin et al. [34,35] describes a primary energy ratio metric that is the ratio of heating provided to primary energy consumed. Use of this metric helps get around the problem of how to treat electricity generated on site. The literature review in this paper focuses on GSHP systems that are characterized with SPF or COPs with the denominator being the electricity consumed without regard to whether it is generated on site or off site.

An aquifer thermal energy storage (ATES) system is a type of multi-well open-loop GSHP system, with hot and cold reservoirs maintained by seasonally switching the extraction and reinjection wells. Schmidt and Müller-Steinhagen [36] described a rather complicated system used to provide heating to a $7000 \mathrm{~m}^{2}$ apartment building. This system combined solar collectors, a gas-fired boiler, ATES, and heat pumps. Seasonal heating COP for the heat pumps only was reported for three years, with an average value of 4.3 .

Urchueguía et al. [37] compared an air-source heat pump system and a ground-source heat pump system serving portions of a university building in Valencia, Spain. Daily and seasonal performance factors corresponding to the SEPEMO H2 and C2 boundaries were reported. For heating, SPFs of $3.5 \pm 0.6$ and $2.0 \pm 0.3$ were measured for the GSHP and ASHP systems, respectively. For cooling, SPFs of $4.3 \pm 0.6$ and $2.7 \pm 0.4$ were measured for the GSHP and ASHP systems, respectively. Montagud et al. [38] present additional data for this system after five years of operation.

Hughes [39-41] utilizes the SEPEMO boundary schemes to determine daily performance factors ( $\mathrm{H} 2$ and $\mathrm{H} 4$ boundaries) and seasonal performance factors (H1, H2, and $\mathrm{H} 4$ boundaries) for $19 \mathrm{GSHP}$ systems serving non-residential buildings in the United Kingdom. All the buildings were part of the British Renewable Heat Incentive (RHI). SPF $\mathrm{H}_{1}$ values for the 19 buildings ranged from 2.36 to 4.64 with a mean value of $3.26, \mathrm{SPF}_{\mathrm{H} 2}$ values ranged from 2.24 to 4.49 with a mean value of 2.95 , and $\mathrm{SPF}_{\mathrm{H} 4}$ ranged from 1.21-4.12, with a mean value of 2.42, based on one year (July 2015-June 2016) of monitoring. 
Table 1. System boundaries for seasonal performance factors (SPF).

\begin{tabular}{|c|c|c|c|c|c|c|c|c|c|}
\hline Reference & $\begin{array}{l}\text { Boundary } \\
\text { Name }\end{array}$ & Heat Pump & $\begin{array}{l}\mathrm{CP} \text { and Fans } \\
\text { on } \\
\text { Source-Side }\end{array}$ & $\begin{array}{l}\text { Auxiliary } \\
\text { Heating }\end{array}$ & $\begin{array}{l}\mathrm{CP} \text { and Fans } \\
\text { on Load-Side } \\
\text { (between HP } \\
\text { and BT) }\end{array}$ & $\begin{array}{c}\text { CP and Fans on } \\
\text { Load-Side (between } \\
\text { BT and Building Heat } \\
\text { Distribution System) }\end{array}$ & $\begin{array}{l}\text { Fan Coil Unit } \\
\text { Fans }\end{array}$ & $\begin{array}{l}\text { Supplementary } \\
\text { Cooling }\end{array}$ & Notes \\
\hline \multirow{8}{*}{ SEPEMO [22] } & H1 & $x$ & & & & & & & \multirow{8}{*}{ No boundary at the buffer tank. } \\
\hline & $\mathrm{H} 2$ & $\mathrm{x}$ & $\mathrm{x}$ & & & & & & \\
\hline & H3 & $\mathrm{x}$ & $\mathrm{x}$ & $\mathrm{x}$ & & & & & \\
\hline & $\mathrm{H} 4$ & $\mathrm{x}$ & $\mathrm{x}$ & $\mathrm{x}$ & $\mathrm{x}$ & $\mathrm{x}$ & & & \\
\hline & $\mathrm{C} 1$ & $\mathrm{x}$ & & & & & & & \\
\hline & $\mathrm{C} 2$ & $\mathrm{x}$ & $\mathrm{x}$ & & & & & & \\
\hline & $\mathrm{C} 3$ & $\mathrm{x}$ & $\mathrm{x}$ & & $x$ & $\mathrm{x}$ & & & \\
\hline & $\mathrm{C} 4$ & $\mathrm{x}$ & $\mathrm{x}$ & & $\mathrm{x}$ & $\mathrm{x}$ & & $\mathrm{x}$ & \\
\hline \multirow{4}{*}{ Winiger et al. [42] } & I & & $x$ & & & & & & \multirow{4}{*}{$\begin{array}{l}\text { Applies to both heating and } \\
\text { cooling; supplementary cooling } \\
\text { not accounted for. }\end{array}$} \\
\hline & II & $\mathrm{x}$ & $x$ & & & & & & \\
\hline & III & $\mathrm{x}$ & $\mathrm{x}$ & $x$ & & & & & \\
\hline & IV & $x$ & $x$ & $\mathrm{x}$ & $x$ & $x$ & & & \\
\hline \multirow{3}{*}{ GroundMed [43] } & I & $x$ & & & & & & & \multirow{3}{*}{$\begin{array}{l}\text { Heating, cooling defined with } \\
\text { same scheme. Supplementary } \\
\text { heating, cooling are not treated. }\end{array}$} \\
\hline & II & $x$ & $x$ & & & & & & \\
\hline & $\begin{array}{l}\text { III } \\
\text { IV }\end{array}$ & $\begin{array}{l}x \\
x\end{array}$ & $\begin{array}{l}x \\
x\end{array}$ & & $\begin{array}{l}x \\
x\end{array}$ & $\begin{array}{l}x \\
x\end{array}$ & $x$ & & \\
\hline \multirow{4}{*}{ Miara et al. [23] } & 0 & $x$ & & & & & & & \multirow{4}{*}{$\begin{array}{l}\text { Only defined for heating. Buffer } \\
\text { tanks for space heating and for } \\
\text { DHW not included. }\end{array}$} \\
\hline & 1 & $\begin{array}{l}x \\
x\end{array}$ & $\mathrm{x}$ & & & & & & \\
\hline & 2 & $\mathrm{x}$ & $\mathrm{x}$ & $\mathrm{x}$ & & & & & \\
\hline & 3 & $\hat{x}$ & $\hat{x}$ & $\hat{x}$ & $x$ & & & & \\
\hline \multirow{4}{*}{ VDI 4650 [25] } & 1 & $x$ & & & & & & & \multirow{4}{*}{ Only defined for heating. } \\
\hline & 2 & & $\mathrm{x}$ & & & & & & \\
\hline & 3 & $x$ & $x$ & $x$ & & & & & \\
\hline & 4 & $x$ & $x$ & $x$ & $x$ & $x$ & & & \\
\hline \multirow{6}{*}{ Koenigsdorff [24] } & $\mathrm{a}^{\prime}$ & $x$ & & & & & & & \multirow{6}{*}{$\begin{array}{l}\text { Only defined for heating. } \\
\text { *Including buffer tank for space } \\
\text { heating, but not for DHW. } \\
\text { **Including buffer tanks for } \\
\text { space heating and for DHW with } \\
\text { in-built electric heater. }\end{array}$} \\
\hline & $a^{\prime \prime}$ & & $\begin{array}{l}x \\
x\end{array}$ & & & & & & \\
\hline & $\begin{array}{l}\mathrm{b} \\
\mathrm{c}\end{array}$ & $\begin{array}{l}x \\
x\end{array}$ & $\begin{array}{l}X \\
X\end{array}$ & $\begin{array}{l}x \\
x\end{array}$ & $\mathrm{X}^{*}$ & & & & \\
\hline & d & $\mathrm{x}$ & $x$ & $x$ & $X^{* *}$ & & & & \\
\hline & $\mathrm{e}$ & $\mathrm{x}$ & $\mathrm{X}$ & $\mathrm{x}$ & $x$ & $x$ & & & \\
\hline & $\mathrm{f}$ & $\mathrm{x}$ & $\mathrm{x}$ & $\mathrm{x}$ & $x$ & $x$ & $\mathrm{x}$ & & \\
\hline
\end{tabular}

X: included. 
Zhai and Yang [44] describe a large (280 boreholes) GSHP system serving a government building housing archives, and hence requiring close control of space air temperature and humidity. Average COPs of the heat pumps (presumably SEPEMO H1 and C1, but not explicitly stated) are given as 4.7 in the summer and 4.6 in the winter.

Vanhoudt et al. [45] describe three years of performance monitoring results for an ATES system. The heat pumps have seasonal performance factors (SEPEMO boundaries H1 and C1) of 5.6 and 5.0. Seasonal system performance factors are also reported, though the boundaries are unclear-it is not clear whether "different circulation pumps" include both source-side and load-side pump energy consumption. Average values of 5.9 and 26.1 are reported. These are higher than the heat pump COPs because some heat is provided directly from the groundwater (presumably for tempering ventilation air) and much of the cooling is provided directly from the groundwater.

Michopoulos et al. [46] monitored a ground-source heat pump system serving an office building in Greece. Weekly and seasonal performance factors corresponding to the SEPEMO H1 and C1 boundary conditions were presented. The seasonal performance factors vary from year-to-year, but are about $5-5.5$ for heating and $4-4.5$ for cooling.

Winiger et al. [42] report on analysis of ten ground-source heat pump systems serving non-residential buildings in Germany. These systems were monitored as part of the broader Energy-Optimized Building (EnOB) project [47]. The authors describe four system boundaries (see Table 1) that have been evaluated, though none of the boundaries include only the heat pump. One case makes use of waste heating, allowing heating without heat pump operation-in this case, the heating SPF I was 61.7. Cooling SPF results are divided between systems using direct cooling (i.e., fluid circulated directly from the ground heat exchanger to the fan coil unit) and active cooling (i.e., using the heat pumps). When heating and cooling were being provided simultaneously, the electricity usage was allocated proportional to the thermal energy provided in each mode. Heating SPF II values (i.e., heat pump plus source-side circulating pump) ranged from 2.9 to 5.7. Heating and cooling SPF IV values (i.e., the entire system including the distribution system) ranged from 1.0-2.7 and 2.2-15.0, respectively, with higher cooling SPFs found for the direct cooling cases. SPF I and SPF II are given for six individual systems, with no other details provided.

Mermoud et al. [48] used the SEPEMO boundary conditions to analyze a GSHP system serving a $2200 \mathrm{~m}^{2}$ office building in Geneva, Switzerland. The system provides heating with heat pumps and direct cooling using the ground heat exchanger fluid. Heat pump COPs are presented for five-minute interval data, showing a significant decrease from the manufacturer's data. The system utilizes two heat pumps and the authors computed separate $\mathrm{SPF}_{\mathrm{H} 1}$ and $\mathrm{SPF}_{\mathrm{H} 2}$, for each heat pump, with values of $\mathrm{SPF}_{\mathrm{H} 1}$ of 3.5 and 3.1 and $\mathrm{SPF}_{\mathrm{H} 2}$ of 3.1 and 3.0. Overall values of $\mathrm{SPF}_{\mathrm{H} 3}$ and $\mathrm{SPF}_{\mathrm{H} 4}$ are 3.1 and 3.0. As the system has favorable return fluid temperatures from the ground (exceeding $10^{\circ} \mathrm{C}$ all year) and the system only provides space heating, the authors find the SPF values disappointing. The poor performance is attributed to the hydraulic design, which requires higher supply water temperatures than what is actually needed.

Monitoring studies of two distributed heat pump systems at the ASHRAE Headquarters building in Atlanta were presented by Southard et al. [49,50] and Spitler et al. [51]. The two systems were an air-source variable-refrigerant flow heat pump system and a ground-source heat pump system. Seasonal heating and cooling system coefficients of performance were calculated for the two systems. Because the systems were distributed and delivered heating and cooling with fans integrated in the heat pump units, and the electrical energy was measured for all heat pumps and the source-side circulating pump together, the coefficients of performance correspond to SEPEMO levels $\mathrm{H} 4$ and C4. The air-source heat pump system had seasonal heating and cooling COPs of $2.0 \pm 0.1$ and $2.5 \pm 0.1$, respectively. The ground-source heat pump system had seasonal heating and cooling COPs of $3.3 \pm 0.2$ and $4.3 \pm 0.6$, respectively.

Spitler et al. [51] presented system heating and cooling COPs as a function of outdoor temperature and heat pump entering fluid temperature. For both the air-source and ground-source heat pump 
systems, cooling COP increased with increasing outdoor air temperature and heat pump entering fluid temperature over a substantial part of the operation range. The counterintuitive behavior of the GSHP system was explained by parasitic losses due to control boards that consume power all the time, fans that run even when all compressors are off, source-side circulating pump energy consumption, and cycling losses of the heat pumps. Recent research [52,53] into cycling losses shows the decreased COP that occurs during the first minute of operation can have a variable but significant effect on heat pump performance.

Mendrinos and Karytsas [43] report on annual heating and cooling SPF for eight GSHP systems in southern Europe monitored as part of the EU Ground-Med project. Additional details can be found in Carvalho et al. [54] and Pardo and Michal [55]. Four levels of system boundaries are defined. As shown in Table 1, these are similar to the SEPEMO boundary conditions for heating, except they do not account for auxiliary heating, and the energy used by the fans in the fan-coil units is treated separately [55] from the load-side circulating pumps. Heating and cooling SPFs for all buildings for all four levels are reported graphically; heating SPF II ranges from 3.6-5.9. Cooling SPF II results are divided between systems using direct cooling (as defined above)-values between 9.8 and 16.6 are achieved, and active cooling-values between 4.9 and 6.8 were achieved.

Schibuola and Scarpa [56] made field measurements of system performance for a ground-source heat pump system providing heating to an $1840 \mathrm{~m}^{2}$ university building originally constructed in the 16th century. Annual heating COPs corresponding to SEPEMO H1 and $\mathrm{H} 2$ were measured at 3.96 and 3.65, respectively. Annual cooling energy efficiency ratios (EERs) corresponding to SEPEMO C1 and C2, were measured at 4.32 and 4.02 , respectively. These results were compared to simulated results for an air-source heat pump system.

Pater and Ciesielczyk [57] compare theoretical performance coefficients provided by the heat pump manufacturer with SPF obtained in real operating conditions for a GSHP installed in a $460 \mathrm{~m}^{2}$ mixed-use building near Krakow in Poland. Measurements were carried out over three heating seasons (September-May). The reported SPF3, according to the Miara et al. [23] system boundary scheme, ranged between 3.4-3.8 over the three heating seasons, which fell within the range of expected SPF based on the manufacturer's technical data for the heat pumps.

Garber-Slaght and Peterson [58] describe a ground-source heat pump system utilizing horizontal ground heat exchangers in a sub-Arctic climate with permafrost present. The permafrost layer at the site was 3 to $7.3 \mathrm{~m}$ deep in 2006 and the slinky heat exchangers are installed at a depth of $2.7 \mathrm{~m}$. The GSHP serves a $446 \mathrm{~m}^{2}$ office space. Monthly heating COPs were measured for three years; an approximate average value of 3.5 may be inferred, with some decrease from the first year to the third year.

Liu et al. [59] summarized annual performance measurements of 10 ground-source heat pump systems serving commercial buildings. The ground-sources included both open loop and closed loop borehole systems, as well as a mine water system and a system utilizing municipal wastewater as the source. Most of the systems used distributed water-to-air heat pumps to provide both heating and cooling. Annual heating and cooling system coefficients of performance (SCOP) were determined for eight of the ten buildings. For the other two, difficulties in differentiating between energy used to provide heating and energy used to provide cooling led the authors to define an effective overall SCOP that combined heating and cooling. Annual heating SCOP varied between about 2.5 and 4.3; annual cooling SCOP varied between about 3 and 5.2. The most commonly identified problem was excessive pumping power caused by oversized circulation pumps, suboptimal controls, and excess hydraulic resistance. For the water-to-air heat pump systems, it was not possible to measure the heating and cooling provided, so a model-based approach was utilized. The resulting uncertainty was not characterized in the studies.

Naicker and Rees [60] present seasonal system performance factors for a GSHP system serving a university building in Leicester, England. Seasonal performance factors are presented for SEPEMO levels $\mathrm{C} 1$ and $\mathrm{H} 1$ (3.19 and 4.06, respectively). Combined cooling and heating SPF are also defined corresponding to SEPEMO levels $\mathrm{H} 1, \mathrm{H} 2$, and $\mathrm{H} 4$ boundary conditions and are referred to as $\mathrm{SPF}_{1}$, 
$\mathrm{SPF}_{2}$, and $\mathrm{SPF}_{4}$ with values of $3.54,2.97,2.49$, respectively. Hourly performance factors are also plotted against cycle time, demonstrating that cycling losses increase with decreasing cycle time. Daily combined performance factors are about 4 under high load conditions, but show considerable scatter at lower daily energy demands leading to an average $\mathrm{SPF}_{1}$ value of 3.54 . SPF2 and SPF4 are affected by the pumping controls that start the circulating pumps three minutes before the compressors. Under low load conditions, with short cycles, the pumping energy consumption can be as high as $30 \%$ of the heat pump energy consumption. Several approaches to improving the system performance are identified, including incorporating buffer tanks, use of a smaller capacity "lead heat pump", and use of variable speed compressors.

Gehlin et al. [61] provide a preliminary analysis of the work described in this paper-analysis of a GSHP system serving a university building in Stockholm, Sweden.

\subsection{Literature Review-Summary}

Long-term measured ( $>1$ year) SPF and COP values reported in the literature for 55 GSHP systems used in commercial or multifamily residential buildings are summarized in Table 2, based on the SEPEMO definitions. In addition, there are three systems for which a combined cooling and heating SPF was given; these appear in the column "HC4". In cases where values were given for multiple years, they have been averaged. The 55 systems shown in Table 2 are sparsely located on three continents. There is a notable inconsistency in which boundaries are used, making comparisons difficult. In a number of cases, the boundaries used are not clearly defined, again making comparisons difficult. Only about half the cases give the overall (H4 or C4) system SPF. Only three of the studies report any uncertainty analysis; only Hughes [41] reports the methodology in detail. Yet this is critical to understanding the significance of the results, so a second contribution in this paper is a detailed description of the uncertainty analysis methodology.

Median values of annual SPF for heating are 4.1, 3.6, 3.1, and 2.9 for boundaries H1, H2, H3, and H4, respectively. Median values of SPF for cooling are 5.5, 6.4, and 4.2 for boundaries C1, C2, and C4, respectively. As noted by other authors [21,22] covering residential GSHP systems, there is a significant range in SPF values that cannot be explained solely by different equipment or different climatic conditions.

Almost all of the past studies provide only seasonal or annual performance factors. But daily performance factors $[59,60]$ or binned average values [51] have been useful in identifying causes for poor performance, such as heat pump cycling losses, pump controls, and equipment failures.

It is the objective of this paper to provide long-term measured system performance data including a detailed uncertainty analysis from a recently installed centralized GSHP system for a new-built office building in the Swedish capital Stockholm. The analysis includes seasonal performance factors and monthly, daily, and binned average values of coefficients of performance. These analyses show how the various system components and controls affect the performance and describe how some unanticipated consequences of the design may be ameliorated. (In the analysis of the Studenthuset GSHP system, we use the "Seasonal Performance Factor (SPF)" to refer to quantities calculated over a year, i.e., 365 sequential days, and "Coefficient of Performance (COP)" for performance factors calculated over shorter periods of time, such as monthly performance). 
Table 2. SPF and coefficient of performance (COP) values for ground source heat pump (GSHP) systems in commercial or multifamily residential buildings.

\begin{tabular}{|c|c|c|c|c|c|c|c|c|c|c|c|c|c|c|c|c|c|c|c|c|c|c|c|}
\hline ID & Reference & Year & Location & $\begin{array}{l}\text { Tot. m of } \\
\text { Borehole }\end{array}$ & $\begin{array}{l}\text { System } \\
\text { Type }^{1}\end{array}$ & $\begin{array}{l}\text { Uncer- } \\
\text { Tainty } \\
\text { Analysis }\end{array}$ & $\underset{2}{\text { Nom. Cap. }}$ & $\begin{array}{l}\text { Nom } \\
\text { W/m }\end{array}$ & $\begin{array}{l}\mathrm{UGT}^{3} \\
\left({ }^{\circ} \mathrm{C}\right)\end{array}$ & $\begin{array}{l}\text { SPF } \\
\text { H1 }\end{array}$ & $\begin{array}{l}\text { SPF } \\
\text { H2 }\end{array}$ & $\begin{array}{l}\text { SPF } \\
\text { H3 }\end{array}$ & $\begin{array}{l}\text { SPF } \\
\text { H4 }\end{array}$ & $\begin{array}{l}\text { SPF } \\
\text { C1 }\end{array}$ & $\begin{array}{l}\text { SPF } \\
\text { C2 }\end{array}$ & $\begin{array}{l}\mathrm{SPF} \\
\mathrm{C} 3\end{array}$ & $\begin{array}{l}\text { SPF } \\
\text { C4 }\end{array}$ & $\begin{array}{l}\text { SPF } \\
\text { HC4 }\end{array}$ & Annual & Monthly & Daily & Hourly & Binned \\
\hline 1 & [58] & 2017 & $\begin{array}{c}\text { USA, } \\
\text { Fairbanks, } \\
\text { AK }\end{array}$ & $\begin{array}{c}\text { Horizontal-1463 } \\
\mathrm{m}\end{array}$ & C & $\mathrm{N}$ & 21 & 14 & 2.4 & $3.5^{*}$ & & & & & & & & & & $x$ & & & \\
\hline 2 & [59] & 2017 & $\begin{array}{c}\text { USA, } \\
\text { Kalispell, MT }\end{array}$ & Ground-water & C & $\mathrm{N}$ & 280 & & 7.7 & & & & 2.7 & & & & & & $x$ & & & & \\
\hline 3 & [59] & 2017 & $\begin{array}{c}\text { USA, } \\
\text { Cedarville, } \\
\text { AR }\end{array}$ & 11,948 & D & $\mathrm{N}$ & 812 & 68 & 16.9 & & & & 3.3 & & & & 3.1 & & $x$ & & $x$ & & \\
\hline 4 & [59] & 2017 & $\begin{array}{c}\text { USA, } \\
\text { Raleigh, NC }\end{array}$ & 6126 & D & N & 300 & 49 & 16.4 & & & & 4.0 & & & & 4.3 & & $x$ & & & & \\
\hline 5 & [59] & 2017 & $\begin{array}{l}\text { USA, Albany, } \\
\text { NY }\end{array}$ & 20,574 & D & $\mathrm{N}$ & 1253 & 61 & 10.0 & & & & 2.5 & & & & 3.0 & & $x$ & & & & \\
\hline 6 & [59] & 2017 & $\begin{array}{c}\text { USA, } \\
\text { Rochester, } \\
\text { MI }\end{array}$ & 24,969 & D & $\mathrm{N}$ & 1540 & 62 & 11.2 & & & & 2.7 & & & & 4.2 & & $x$ & & & & \\
\hline 7 & [59] & 2017 & $\begin{array}{l}\text { USA, Butte, } \\
\text { MT }\end{array}$ & Mine water & C & $\mathrm{N}$ & 175 & & 8.1 & & & & 3.7 & & & & 3.6 & & $x$ & & & & \\
\hline 8 & [59] & 2017 & $\begin{array}{c}\text { USA, } \\
\text { Greenville, } \\
\text { SC }\end{array}$ & 30,480 & D & $\mathrm{N}$ & 2153 & 71 & 19.5 & & & & 4.4 & & & & 5.2 & & $x$ & & & & \\
\hline 9 & [59] & 2017 & $\begin{array}{l}\text { USA, Denver, } \\
\text { CO }\end{array}$ & Waste water & C & N & 754 & & 11.6 & & & & & & & & & 3.7 & $x$ & & & & \\
\hline 10 & [59] & 2017 & $\begin{array}{c}\text { USA, } \\
\text { Lincoln, NE }\end{array}$ & 60,990 & C & $\mathrm{N}$ & 4900 & 80 & 11.6 & & & & 3.6 & & & & 5.3 & & $x$ & & & & \\
\hline 11 & [59] & 2017 & $\begin{array}{c}\text { USA, } \\
\text { Muncie, IN }\end{array}$ & 219,822 & C & $\mathrm{N}$ & 17,500 & 80 & 12.4 & & & & & & & & & 3.7 & $x$ & & & & \\
\hline 12 & [43] & 2016 & $\begin{array}{l}\text { Greece, } \\
\text { Athens } \\
\end{array}$ & 1200 & C & $\mathrm{N}$ & 55 & 46 & 20.0 & 6.4 & 5.6 & & 2.9 & 6.3 & 5.5 & & 2.7 & & $x$ & & & & \\
\hline 13 & [43] & 2016 & $\begin{array}{c}\text { Spain, } \\
\text { Barcelona }\end{array}$ & 1400 & C & $\mathrm{N}$ & 60 & 43 & 18.0 & 6.4 & 5.9 & & 3.7 & 7.4 & 6.8 & & 3.3 & & $x$ & & & & \\
\hline 14 & [43] & 2016 & $\begin{array}{l}\text { Slovenia, } \\
\text { Benedikt }\end{array}$ & 390 & C & $\mathrm{N}$ & 20 & 52 & 11.1 & 5.6 & 5.3 & & 4.7 & & 9.8 & & 7.0 & & $x$ & & & & \\
\hline 15 & [43] & 2016 & $\begin{array}{l}\text { Portugal, } \\
\text { Coimbra } \\
\end{array}$ & 875 & C & $\mathrm{N}$ & 70.4 & 80 & 17.5 & 5.4 & 5.2 & & 4.3 & 6.8 & 6.0 & & 4.0 & & $x$ & & & & \\
\hline 16 & [43] & 2016 & $\begin{array}{c}\text { France, } \\
\text { Septèmes les } \\
\text { Vallons }\end{array}$ & 600 & C & $\mathrm{N}$ & 26 & 43 & 17.0 & 4.6 & 4.0 & & 3.1 & & 10.5 & & 4.2 & & $x$ & & & & \\
\hline 17 & [43] & 2016 & $\begin{array}{c}\text { Romania, } \\
\text { Oradea }\end{array}$ & 1300 & C & $\mathrm{N}$ & 37 & 28 & 12.0 & 5.4 & 5.0 & & 4.4 & 7.3 & 6.7 & & 5.1 & & $x$ & & & & \\
\hline 18 & {$[43]$} & 2016 & Italy, Padova & 320 & $\mathrm{C}$ & $\mathrm{N}$ & 14 & 45 & 15.0 & 4.1 & 3.6 & & 3.2 & 5.6 & 5.1 & & 4.2 & & $x$ & & & & \\
\hline 19 & [43] & 2016 & $\begin{array}{c}\text { Spain, } \\
\text { Valencia }\end{array}$ & 300 & C & $\mathrm{N}$ & 18 & 60 & 17.7 & 4.7 & 4.5 & & 2.9 & 5.3 & 4.8 & & 2.9 & & $x$ & & & & \\
\hline
\end{tabular}


Table 2. Cont

\begin{tabular}{|c|c|c|c|c|c|c|c|c|c|c|c|c|c|c|c|c|c|c|c|c|c|c|c|}
\hline ID & Reference & Year & Location & $\begin{array}{l}\text { Tot. } m \text { of } \\
\text { Borehole }\end{array}$ & $\begin{array}{l}\text { System } \\
\text { Type }^{1}\end{array}$ & $\begin{array}{c}\text { Uncer- } \\
\text { Tainty } \\
\text { Analysis }\end{array}$ & $\begin{array}{l}\text { Nom. Cap. } \\
{ }_{(\mathrm{kW})}\end{array}$ & $\begin{array}{l}\text { Nom } \\
\mathrm{W} / \mathrm{m}\end{array}$ & $\begin{array}{l}\text { UGT }^{3} \\
\left({ }^{\circ} \mathrm{C}^{\circ}\right)\end{array}$ & $\begin{array}{l}\text { SPF } \\
\text { H1 }\end{array}$ & $\begin{array}{l}\text { SPF } \\
\text { H2 }\end{array}$ & $\begin{array}{l}\text { SPF } \\
\text { H3 }\end{array}$ & $\begin{array}{l}\text { SPF } \\
\text { H4 }\end{array}$ & $\begin{array}{l}\text { SPF } \\
\text { C1 }\end{array}$ & $\begin{array}{l}\text { SPF } \\
\text { C2 }\end{array}$ & $\begin{array}{l}\text { SPF } \\
\text { C3 }\end{array}$ & $\begin{array}{l}\text { SPF } \\
\text { C4 }\end{array}$ & $\begin{array}{l}\text { SPF } \\
\text { HC4 }\end{array}$ & Annual & Monthly & Daily & Hourly & Binned \\
\hline 20 & [48] & 2014 & $\begin{array}{l}\text { Switzerland, } \\
\text { Geneva }\end{array}$ & 2700 & C & $\mathrm{N}$ & 240 & 89 & 11.7 & & & 3.1 & 3.0 & & & & & & $x$ & & & & \\
\hline 21 & [46] & 2013 & $\begin{array}{l}\text { Greece, } \\
\text { Pylaia }\end{array}$ & 1680 & c & $\mathrm{N}$ & 276 & 164 & 16.5 & 4.9 & & & & 4.5 & & & & & $x$ & & & & \\
\hline 22 & [60] & 2018 & $\begin{array}{l}\text { United } \\
\text { Kingdom, } \\
\text { Leicester }\end{array}$ & 5600 & C & $\mathrm{N}$ & 480 & 86 & 10.6 & 4.1 & & & & 3.2 & & & & 2.5 & $x$ & & $x$ & $x$ & \\
\hline 23 & [57] & 2017 & $\begin{array}{l}\text { Poland, } \\
\text { Cracow }\end{array}$ & 210 & C & $\mathrm{N}$ & 10 & 48 & 9.9 & & & $3.6^{*}$ & & & & & & & $x$ & & & & \\
\hline 24 & [56] & 2016 & Italy, Venice & 720 & C & $\mathrm{N}$ & 50 & 69 & 14.9 & 4.0 & 3.7 & & & 4.3 & 4.0 & & & & $x$ & $x$ & & & \\
\hline 25 & [36] & 2004 & $\begin{array}{l}\text { Germany, } \\
\text { Rostock }\end{array}$ & ATES & c & $\mathrm{N}$ & & & 9.8 & 4.3 & & & & & & & & & $x$ & & & & \\
\hline 26 & [49-51] & 2014 & $\begin{array}{l}\text { USA, Atlanta, } \\
\text { GA }\end{array}$ & 1464 & D & Y & 111 & 76 & 17.1 & & & & 3.3 & & & & 4.3 & & $x$ & $x$ & & & $x$ \\
\hline 27 & [37] & 2008 & $\begin{array}{c}\text { Spain, } \\
\text { Valencia }\end{array}$ & 300 & C & Y & 17 & 57 & 17.1 & & 3.5 & & & 4.3 & & & & & $x$ & & $x$ & & \\
\hline 28 & [45] & 2011 & $\begin{array}{c}\text { Belgium, } \\
\text { Brasschaat }\end{array}$ & ATES & C & $\mathrm{N}$ & 195 & & 11.4 & 5.6 & 5.9 & & & 6.0 & 26.1 & & & & $x$ & & & & \\
\hline 29 & [33] & 2017 & $\begin{array}{l}\text { Switzerland, } \\
\text { Uster }\end{array}$ & 869 & C & $\mathrm{N}$ & 33 & 38 & 10.8 & & 3.9 & & & & & & & & $x$ & & $x$ & & \\
\hline 30 & [42] & 2013 & Germany & & & $\mathrm{N}$ & 270 & & & 3.5 & 3.0 & & & & & & & & $x$ & & & & \\
\hline 31 & [42] & 2013 & Germany & & & $\mathrm{N}$ & 54 & & & 4.9 & 3.6 & & & & & & & & $x$ & & & & \\
\hline 32 & [42] & 2013 & Germany & & & $\mathrm{N}$ & 57 & & & 5.2 & 3.6 & & & & & & & & $x$ & & & & \\
\hline 33 & [42] & 2013 & Germany & & & $\mathrm{N}$ & 122 & & & 5.7 & & & & & & & & & $x$ & & & & \\
\hline 34 & [42] & 2013 & Germany & & & $\mathrm{N}$ & 68 & & & 4.4 & 4.1 & & & & & & & & $x$ & & & & \\
\hline 35 & [42] & 2013 & Germany & & & $\mathrm{N}$ & 75 & & & 6.2 & 5.7 & & & & & & & & $x$ & & & & \\
\hline 36 & [44] & 2011 & $\begin{array}{c}\text { China, } \\
\text { Shanghai }\end{array}$ & 22,400 & C & $\mathrm{N}$ & 1000 & 45 & 18.5 & 4.6 & & & & 4.7 & & & & & $x$ & & & & \\
\hline 37 & [40] & 2018 & $\begin{array}{c}\text { United } \\
\text { Kingdom }\end{array}$ & Ground-water & C & $\mathrm{Y}$ & 26 & & & 4.64 & 4.49 & & 4.12 & & & & & & $x$ & & $x$ & & \\
\hline 38 & [40] & 2018 & $\begin{array}{l}\text { United } \\
\text { Kingdom }\end{array}$ & $\begin{array}{l}\text { Horizontal } \\
2400 \mathrm{~m}\end{array}$ & C & Y & 93 & 38.75 & & 3.76 & 3.44 & & 2.86 & & & & & & $x$ & & $x$ & & \\
\hline 39 & [40] & 2018 & $\begin{array}{c}\text { United } \\
\text { Kingdom }\end{array}$ & $\begin{array}{c}\text { Horizontal } \\
1200 \mathrm{~m}\end{array}$ & C & Y & 21.4 & 17.8 & & 3.98 & 3.42 & & 3.17 & & & & & & $x$ & & $x$ & & \\
\hline 40 & [40] & 2018 & $\begin{array}{c}\text { United } \\
\text { Kingdom }\end{array}$ & Water & C & $\mathrm{Y}$ & 96 & & & 3.11 & 2.72 & & 1.49 & & & & & & $x$ & & $x$ & & \\
\hline 41 & [40] & 2018 & $\begin{array}{c}\text { United } \\
\text { Kingdom }\end{array}$ & $\begin{array}{l}\text { Horizontal } \\
800 \mathrm{~m}\end{array}$ & C & $\mathrm{Y}$ & 22 & 27.5 & & 2.36 & 2.24 & & 1.83 & & & & & & $x$ & & $x$ & & \\
\hline 42 & [40] & 2018 & $\begin{array}{c}\text { United } \\
\text { Kingdom }\end{array}$ & $\begin{array}{l}\text { Horizontal } \\
4000 \mathrm{~m}\end{array}$ & c & $\mathrm{Y}$ & 144 & 36 & & 2.55 & 2.24 & & 1.21 & & & & & & $x$ & & $x$ & & \\
\hline
\end{tabular}


Table 2. Cont.

\begin{tabular}{|c|c|c|c|c|c|c|c|c|c|c|c|c|c|c|c|c|c|c|c|c|c|c|}
\hline ID & Reference & Year & Location & $\begin{array}{l}\text { Tot. m of } \\
\text { Borehole }\end{array}$ & $\begin{array}{l}\text { System } \\
\text { Type }^{1}\end{array}$ & $\begin{array}{c}\text { Uncer- } \\
\text { Tainty } \\
\text { Analysis } \\
\end{array}$ & $\begin{array}{l}\text { Nom. Cap. } \\
2(\mathbf{k} W)\end{array}$ & $\begin{array}{l}\text { Nom } \\
W / m\end{array}$ & $\begin{array}{l}\text { UGT }^{3} \\
\left({ }^{\circ} \mathrm{C}\right)\end{array}$ & $\begin{array}{l}\text { SPF } \\
\text { H1 }\end{array}$ & $\begin{array}{l}\text { SPF } \\
\text { H2 }\end{array}$ & $\begin{array}{l}\text { SPF } \\
\text { H3 }\end{array}$ & $\begin{array}{l}\text { SPF } \\
\mathrm{H} 4\end{array}$ & $\begin{array}{l}\text { SPF } \\
\text { C1 }\end{array}$ & $\begin{array}{l}\text { SPF } \\
\text { C2 }\end{array}$ & $\begin{array}{l}\text { SPF } \\
\text { C3 }\end{array}$ & $\begin{array}{l}\text { SPF } \\
\text { C4 }\end{array}$ & $\begin{array}{l}\text { SPF } \\
\text { HC4 }\end{array}$ & Annual Monthly & Daily & Hourly & Binned \\
\hline 43 & [40] & 2018 & $\begin{array}{c}\text { United } \\
\text { Kingdom }\end{array}$ & Ground-water & C & Y & 60 & & & 3.46 & 2.43 & & 2.14 & & & & & & $x$ & $x$ & & \\
\hline 44 & [40] & 2018 & $\begin{array}{c}\text { United } \\
\text { Kingdom }\end{array}$ & 1500 & C & Y & 54 & 36 & & 2.88 & 2.76 & & 2.54 & & & & & & $x$ & $x$ & & \\
\hline 45 & [40] & 2018 & $\begin{array}{c}\text { United } \\
\text { Kingdom }\end{array}$ & 1500 & C & Y & 70.8 & 47.2 & & 2.95 & 2.73 & & 2.23 & & & & & & $x$ & $x$ & & \\
\hline 46 & [40] & 2018 & $\begin{array}{c}\text { United } \\
\text { Kingdom }\end{array}$ & Coils in river & C & Y & 126 & & & 3.14 & 2.88 & & 2.53 & & & & & & $x$ & $x$ & & \\
\hline 47 & [40] & 2018 & $\begin{array}{c}\text { United } \\
\text { Kingdom } \\
\end{array}$ & Horizontal & C & Y & 14 & & & 4.11 & 3.89 & & 3.21 & & & & & & $x$ & $x$ & & \\
\hline 48 & [40] & 2018 & $\begin{array}{c}\text { United } \\
\text { Kingdom }\end{array}$ & & C & Y & 64 & & & 3.2 & 2.56 & & 2.31 & & & & & & $\mathrm{x}$ & $\mathrm{x}$ & & \\
\hline 49 & [40] & 2018 & $\begin{array}{c}\text { United } \\
\text { Kingdom }\end{array}$ & 575 & C & Y & 19.8 & 34.4 & & 2.7 & 2.38 & & 1.61 & & & & & & $x$ & $x$ & & \\
\hline 50 & [40] & 2018 & $\begin{array}{c}\text { United } \\
\text { Kingdom } \\
\end{array}$ & $\begin{array}{l}\text { Horizontal } \\
1200 \mathrm{~m}\end{array}$ & C & Y & 22.9 & 19.1 & & 3.22 & 3.95 & & 2.96 & & & & & & $x$ & $x$ & & \\
\hline 51 & [40] & 2018 & $\begin{array}{c}\text { United } \\
\text { Kingdom }\end{array}$ & & C & Y & 38.3 & & & 2.85 & 2.69 & & 2.49 & & & & & & $\mathrm{x}$ & $x$ & & \\
\hline 52 & [40] & 2018 & $\begin{array}{c}\text { United } \\
\text { Kingdom } \\
\end{array}$ & River water & c & Y & 30 & & & 3.15 & 2.61 & & 2.22 & & & & & & $\mathrm{x}$ & $x$ & & \\
\hline 53 & [40] & 2018 & $\begin{array}{c}\text { United } \\
\text { Kingdom } \\
\end{array}$ & $\begin{array}{c}\text { Horizontal } \\
1500 \mathrm{~m} \\
\end{array}$ & C & Y & 40 & 26.7 & & 3.21 & 2.88 & & 2.73 & & & & & & $x$ & $\mathrm{x}$ & & \\
\hline 54 & [40] & 2018 & $\begin{array}{c}\text { United } \\
\text { Kingdom }\end{array}$ & 800 & C & Y & 40 & 50 & & 3.48 & 3.15 & & 2.39 & & & & & & $x$ & $x$ & & \\
\hline 55 & [40] & 2018 & $\begin{array}{c}\text { United } \\
\text { Kingdom }\end{array}$ & Open water & c & Y & 268 & & & 3.1 & 2.62 & & 1.99 & & & & & & $x$ & $\mathrm{x}$ & & \\
\hline
\end{tabular}

1 Centralized (C) or distributed (D). 2 Nominal heat pump capacities for either heating or cooling. In a number of cases, the heat pump capacity is not given and is estimated from the floor area and the specific heating or cooling load. 3 Undisturbed ground temperatures (UGT) are taken from the nearest location found in a world-wide database [62]. These values do not include local disturbances due to urbanization. Table entries with UGT left blank correspond to references for which specific locations are not given. * SPF values have been estimated from the authors' presented results. 


\section{System Description}

Stockholm University is located within the large campus area Frescati in central Stockholm. In 2011 the government-owned real-estate owner Akademiska Hus, responsible for management and development of buildings for research and higher education in Sweden, built a new student center at the campus (Figure 1). The new building was completed in the fall of 2013. Ambitions were high for the student center building to be a model building regarding sustainability, ecologic footprint, and energy use. The $6300 \mathrm{~m}^{2}$ four-story building contains office area for 130 staff, small meeting rooms, 200 study-booths for students, and a café, and was designed to be highly energy efficient with a planned total use of $25 \mathrm{kWh} / \mathrm{m}^{2} /$ year ( $<160 \mathrm{MWh} /$ year) bought energy (electricity).

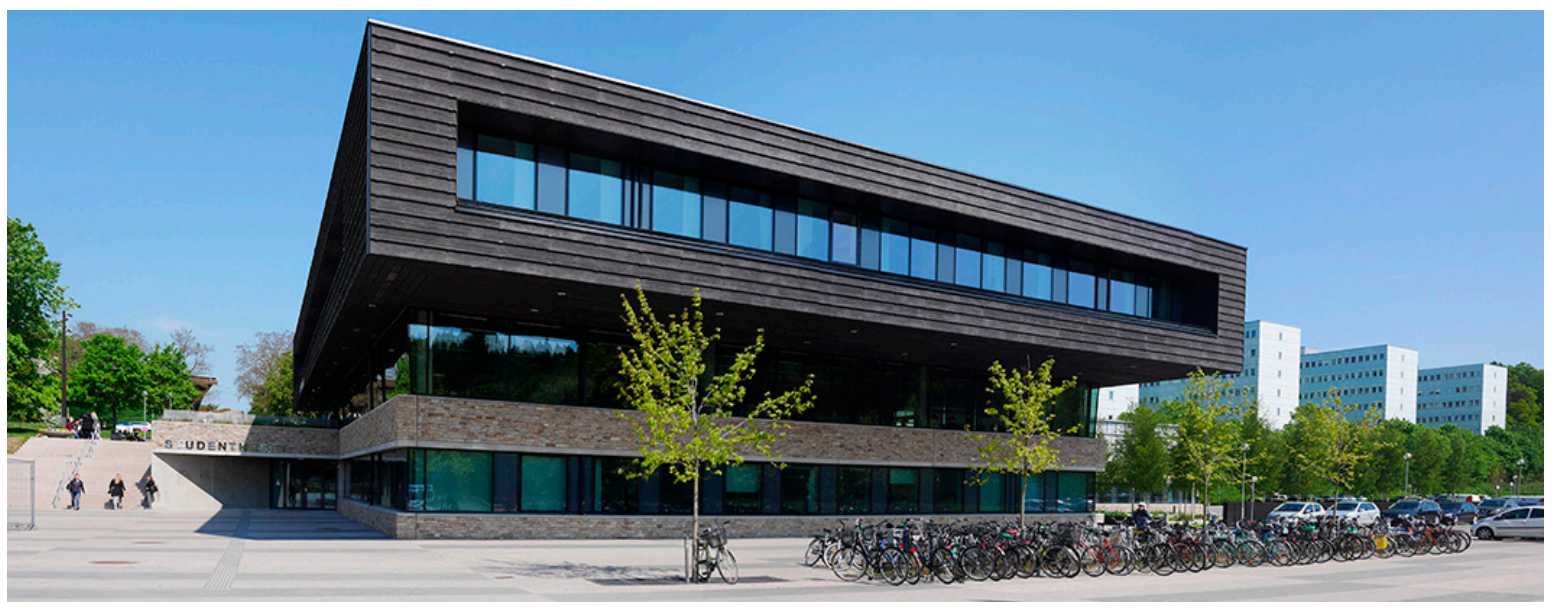

Figure 1. View of Studenthuset. Photo credit: Jeffrey D Spitler.

The building services are thoroughly instrumented. Space heating and domestic hot water (DHW) are provided by a ground source heat pump (GSHP) system consisting of five $40 \mathrm{~kW}$ off-the-shelf water-to-water heat pumps connected to a borehole field. The DHW temperature is further increased by a Legionella protection system consisting of an electric resistance heater that raises the hot water temperature from $55^{\circ} \mathrm{C}$ to $60{ }^{\circ} \mathrm{C}$ and a pump that continuously recirculates hot water around the building. As will be seen later, this has a significant effect on the energy usage of the system. Space cooling is provided by direct cooling from the boreholes; with the maximum fluid temperature leaving the boreholes not to exceed $16^{\circ} \mathrm{C}$. Building occupancy during the warmest summer months (July-August) is low, while the students are on summer vacation. Measured temperatures from the boreholes do not exceed $13.4{ }^{\circ} \mathrm{C}$ during the measurement period, and dehumidification loads are not high. The bore field consists of 20 groundwater-filled boreholes in hard rock, drilled to a depth of $200 \mathrm{~m}$, and fitted with single u-tubes filled with an ethanol/water mixture. The bore field is located below a landscaped courtyard, and the boreholes are drilled at an angle so that they reach under the surrounding building.

Heat distribution inside the building is provided by radiators with a larger-than-usual surface area so that the distribution temperature is $40^{\circ} \mathrm{C}$ instead of $55^{\circ} \mathrm{C}$, which is more common in Sweden. Cooling distribution is done by the ventilation system and chilled beams. The system also includes heat recovery from the kitchen cooling circuit. A schematic of the Studenthuset heating and cooling system is seen in Figure 2.

Prior to building operation, total annual energy loads were anticipated to be $200 \mathrm{MWh}$ heating and $34 \mathrm{MWh}$ cooling. The installed capacity is $200 \mathrm{~kW}$ heating and $120 \mathrm{~kW}$ cooling from the boreholes. No auxiliary heating or cooling is installed, except for the electric resistance heater that boosts the hot water temperature to protect against Legionella. The anticipated electricity use for the building installations was approximately $14 \mathrm{kWh} / \mathrm{m}^{2} /$ year. Distribution losses in the heating system were 
estimated to be $2 \mathrm{kWh} / \mathrm{m}^{2} /$ year. Heat pump SPF was expected to be 4.5 according to the design documents, though the SPF boundaries were not defined.

The climate in Stockholm is characterized by a humid continental climate, with average temperatures of around $-3{ }^{\circ} \mathrm{C}$ in the winter and $+20^{\circ} \mathrm{C}$ in the summer.

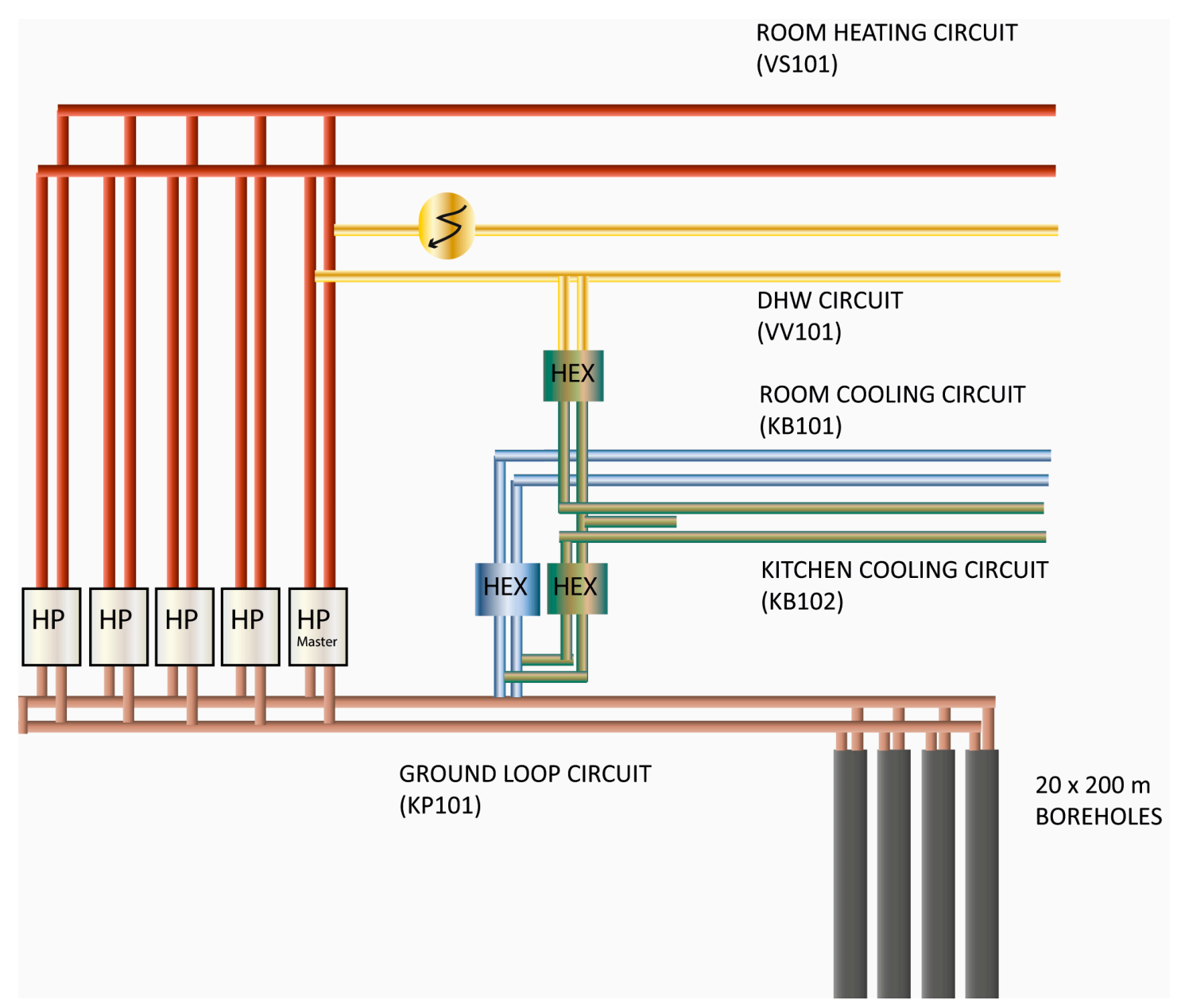

Figure 2. Schematic of the Studenthuset heating and cooling system. Illustration by S. Gehlin.

\section{Measurements and Uncertainty Analysis}

Measurement of the system performance relies on instrumentation installed (mostly) at the same time as the system installation for the owner's benefit in monitoring the system's performance. Some additional instrumentation was installed later to improve the capability to measure the cooling provided and/or estimate the source-side circulating pump energy consumption. Because the instrumentation system was not specifically designed to support measurement of SPFs and COPs with the SEPEMO boundaries, some necessary approximations are described in this section.

\subsection{Instrumentation and Analysis}

As discussed above, the system performance can be measured with different boundary conditions. The system is extensively instrumented compared to many buildings, but, unfortunately, there are no measurements made of airflow rates, so it is not possible to estimate either the heating or cooling provided to the ventilation air or the building by the heat recovery systems. This limits the possibilities for calculating SPFs and COPs with the $\mathrm{H} 4, \mathrm{C} 3$, and $\mathrm{C} 4$ boundaries. Nor are there independent measurements of compressor energy consumption, so SPFs and COPs cannot be calculated for the H1 and $\mathrm{C} 1$ boundaries. 


\subsubsection{Instrumentation and Analysis-Heat Transfer Rates}

With the existing measurements, it is possible to estimate SPFs and COPs for the $\mathrm{C} 2, \mathrm{H} 2$, and $\mathrm{H} 3$ boundaries, and that is the focus of this paper. To measure the system performance with these boundaries, it is necessary to measure the heat transfer rates:

1. from the heat pumps to the building heat distribution system,

2. from the heat pumps to the DHW,

3. from the auxiliary heating system to the DHW

4. from the auxiliary heating system to the building,

5. for cooling, from the building cooling distribution system to the ground loop (some of the heat is dissipated in the boreholes and some is used as a heat source for the heat pumps). This is synonymous with the building cooling provided.

Heat transfer from the heat pumps to the building heat distribution system is measured with an energy meter that determines the heat transfer rate calorimetrically. The energy meter measures the volume flow rate to an accuracy of $\pm 5 \%$ or better. It measures the temperature difference with a matched pair of platinum RTDs (Resistance Temperature Detector), with maximum discrepancy of $0.05 \mathrm{~K}$ and median discrepancy of $0.02 \mathrm{~K}$. The meter reports cumulative energy consumption, but knowledge of the flow meter and temperature sensor accuracies is needed to estimate the uncertainty in the energy consumption reported by the meter.

For purposes of determining the heat provided by the heat pumps to the DHW, only the volume flow rate is measured with a flow meter with $\pm 5 \%$ or better accuracy. The temperature provided by the heat pumps is controlled to $55^{\circ} \mathrm{C}$. The incoming temperature from the Stockholm water supply is not measured; therefore, it was estimated based on measurements made at seven different buildings in Stockholm $[63,64]$. The incoming temperature varies over the year and from location to location. A heuristic equation to describe the incoming water temperature $\left(\right.$ in ${ }^{\circ} \mathrm{C}$ ) was developed:

$$
T_{w, i}=10+5 \cdot \sin \left(\left(\frac{3}{2} \pi\right)+\frac{\left(n-n_{\min }\right)}{365} \cdot(2 \pi)\right)
$$

where $n=$ day of the year; $365 \geq n \geq 1 ; n_{\min }=$ day of the year with minimum temperature (day 60)

The uncertainty for this approximation is estimated as $\pm 2.5^{\circ} \mathrm{C}$. The average heating energy $(\mathrm{kWh})$ provided by the heat pumps to the domestic hot water for any time interval is then given by:

$$
Q_{D H W}=V \rho c_{p}\left(T_{w, \mathrm{OHP}}-T_{w, i}\right)
$$

where $V=$ integrated volume flow over the time interval, $\mathrm{m}^{3} ; \rho=$ density of the water, $\mathrm{kg} / \mathrm{m}^{3} ; c_{p}$ $=$ specific heat of the water, $\mathrm{kJ} /(\mathrm{kg} \cdot \mathrm{K}) ; T_{w, \mathrm{OHP}}=$ temperature of heated water at outlet from heat pumps, ${ }^{\circ} \mathrm{C}$.

Heating provided by the Legionella protection system is estimated based on two contributions: (1) a steady consumption of $3 \mathrm{~kW}$ of electricity due to the recirculation pump and dissipated energy from the hot water that was continuously circulated around the building, and (2) energy provided by the electric resistance heater to heat the DHW from $55^{\circ} \mathrm{C}$ to $60{ }^{\circ} \mathrm{C}$ :

$$
Q_{L P S R H}=V \rho c_{p}\left(T_{w, \mathrm{ORH}}-T_{w, \mathrm{OHP}}\right)
$$

where $V=$ integrated volume flow over the time interval, $\mathrm{m}^{3} ; T_{w, \mathrm{ORH}}=$ temperature of heated water at outlet from resistance heater, ${ }^{\circ} \mathrm{C}$.

The electric meter measuring the heat pumps, hot water recirculation pump, and the electric resistance heater measures $3 \mathrm{~kW}$ of electricity for many hours during the year when there is no domestic hot water use and the heat pumps are not running. This $3 \mathrm{~kW}$ corresponds to the energy consumed by the pump plus the energy dissipated by the recirculation of hot water and which is made up by the 
resistance heater operating. For these hours, the heat is dissipated in the building. This heat may be useful or it may form part of the cooling load. As a rough attempt to distinguish between the two possibilities, we deemed the heat dissipated useful if the building had a heating load.

The cooling provided by the system is measured with the same type of instrumentation as used for measuring the building heating provided.

\subsubsection{Instrumentation and Analysis-Electrical Energy}

In addition to estimating the various heat transfer rates, it is also necessary to measure the electrical energy used by the heat pumps, source-side circulating pump(s), and the auxiliary heating system. The system electrical energy consumption is monitored continuously and recorded on an hourly basis.

One meter logs electricity use for the five heat pumps (compressors and built-in circulation pumps), including the heat pump that is dedicated to DHW heating. It also includes electricity consumed by the Legionella protection system. This meter has an accuracy of $\pm 1 \%$. In order to estimate the electrical energy consumed by the heat pumps for boundary $\mathrm{H} 2$, it is necessary to be able to subtract out the energy consumed by the Legionella protection system.

As discussed above, electrical power for the recirculation pump is essentially constant over the year at a level of $3 \mathrm{~kW}$. Electrical energy for the electric resistance heater can be estimated calorimetrically using Equation (3). These values are subtracted from the meter measurements to obtain the electrical energy consumed by the heat pumps.

Electrical energy consumed by the source-side circulation pump was metered along with the electrical energy for fans used for air conditioning, circulation pumps on the load-side (distribution), and circulation pumps on the source-side (boreholes), as well as electricity used for running the rotary exhaust air heat exchangers in the kitchen and building. However, for purposes of our analysis, it was necessary to be able to separately estimate the electrical energy consumed by the source-side circulation pump. This variable speed pump is controlled to maintain a minimum flow rate of $8 \mathrm{~L} / \mathrm{s}$. A separate set of measurements over a two-week period was made to allow estimation of the electricity $(\mathrm{kW})$ used by the source-side circulation pump as a function of flow rate with this equation:

$$
P_{S S C P}=0.001973 \dot{V}^{3}
$$

where $\dot{V}=$ volume flow rate averaged over the time interval, L/s.

The resulting power given by Equation (4) is estimated to have an uncertainty of $\pm 0.19 \mathrm{~kW}$.

A further complication arises for the source-side circulation pump-for many, many hours of operation, both heating and cooling are being provided by the system. In this case, some of the electrical energy should be allocated to the heating electrical energy and some should be allocated to the cooling electrical energy. How should this allocation be done? We chose to allocate it based on the amount of heating and cooling provided, without regard to the fact that the heating is provided via heat pumps, panel radiators, and fan coil units, while the cooling is provided "directly" with fan coil units and chilled beams. The heating provided does not include incidental heating provided by the Legionella protection system. Specifically, the fraction of electrical energy consumed by the circulating pump that is allocated to the heating electrical energy is determined as:

$$
f_{h t g}=\frac{q_{B l d g H t g}+q_{D H W}}{q_{\text {BldgHtg }}+q_{D H W}+q_{\text {BldgClg }}}
$$

where $q_{B l d g H t g}=$ average heating provided to the building over the time interval, $\mathrm{kW} ; q_{D H W}=$ average heating provided to the domestic hot water over the time interval, $\mathrm{kW} ; q_{B l d g C l g}=$ average cooling provided to the building over the time interval, $\mathrm{kW}$.

The fraction of the electrical energy consumed by the circulating pump allocated to cooling is then:

$$
f_{c l g}=1-f_{h t g}
$$


The scheme used to allocate the electrical energy consumed by the circulating pump is admittedly arbitrary, but a better scheme has not, to the authors' knowledge, been suggested in the literature. A similar approach was used by Winiger et al. [42].

\subsection{Uncertainty Analysis}

It is highly desirable to estimate the uncertainty of the quantities of interest, i.e., COPs, for several reasons. These include understanding the significance of the results-e.g., are the results meaningful; are trends in the results meaningful? The uncertainty analysis can also shed light on the design and specification of instrumentation for future monitoring projects.

The uncertainty analysis, which involves calculation of the propagation of uncertainties from the physical measurements to the final quantities of interest, follows the general procedures described by Taylor [65]. Like Taylor, we use the words "error" and "uncertainty" interchangeably.

To set the context, the final quantities of interest are primarily coefficients of performance over some time period —annual, monthly—or over all times in a specific outdoor air temperature bin or entering fluid temperature bin. So, while random errors exist, they are usually mitigated by a large number of measurements over which a specific COP value is computed. One exception is COPs computed for bins with very few hours, e.g., less than 10 measured hours. Accordingly, systematic errors are of more concern and random errors are neglected in this uncertainty analysis.

\subsubsection{Uncertainty Analysis-Heating and Cooling Provided}

The building heating and cooling are both measured with energy meters that incorporate a flow meter with $\pm 5 \%$ accuracy and a matched pair of temperature sensors that are selected to have a discrepancy (i.e., measurement error relative to one another) of no more than $0.05 \mathrm{~K}$. Therefore, the individual uncertainties can be expressed:

$$
\begin{gathered}
e_{\text {flow }}=0.05 \\
E_{\Delta T}=0.05 \mathrm{~K} \\
e_{\Delta T}=\frac{0.05 \mathrm{~K}}{\Delta T}
\end{gathered}
$$

(We adopt the convention here that fractional uncertainties are expressed with a lower-case " $e$ " and absolute uncertainties given in the units of the quantity in question are expressed with an upper-case " $E$ ". If the quantity in question is " $x$ ", $E_{x}=x \cdot e_{x}$.) Since errors in the flow rate and temperature difference measurement are independent of each other, they can be added in quadrature to determine the uncertainty of the heat transfer rate at each hour.

$$
e_{q}=\sqrt{e_{\text {flow }}^{2}+e_{\Delta T}^{2}}
$$

Since the errors in determining the density and specific heat are relatively small compared to the volume flow rate uncertainty, these errors are neglected. Over the one-year period, the uncertainty in the building heating provided has an average value of $\pm 6 \%$ and a maximum value of $\pm 11 \%$. Over the same period, the uncertainty in the building cooling provided has an average value $\pm 5 \%$ and a maximum value of $\pm 26 \%$. The maximum values occur for hours with very low temperature differences and hence low heat transfer rates.

These hourly uncertainties are aggregated for time periods or bins by summing the absolute uncertainties, e.g., to find the uncertainty in building heating provided for a one-month period, with $720 \mathrm{~h}$ :

$$
E_{q, \text { month }}=\sum_{i=1}^{720} E_{q, i}
$$




$$
e_{q, \text { month }}=\frac{E_{q, \text { month }}}{\sum_{i=1}^{720} q_{i}}
$$

The uncertainty in the heating provided by the heat pumps to the domestic hot water is calculated similarly to that of the building heating and cooling, but the uncertainty in the temperature difference is somewhat higher. The uncertainty for the inlet temperature (Equation (1)) is taken as $\pm 2.5^{\circ} \mathrm{C}$, and the uncertainty for the outlet temperature is taken as $\pm 1^{\circ} \mathrm{C}$. The two errors are independent, so the uncertainty in the temperature difference may be taken as:

$$
\begin{gathered}
E_{\Delta T}=\sqrt{2.5^{2}+1^{2}}=2.7^{\circ} \mathrm{C} \\
e_{\Delta T}=\frac{2.7^{\circ} \mathrm{C}}{\Delta T}
\end{gathered}
$$

Equation (10) is used to estimate the final uncertainty in the heating provided by the heat pumps to the domestic hot water. Although the uncertainty in the temperature difference is higher than that for the building heating and cooling, the temperature difference is significantly higher, so the average uncertainty is $\pm 8 \%$ and the maximum (when rounded to the nearest $\%$ ) is also $\pm 8 \%$.

Calculation of uncertainty for the DHW heating provided by the Legionella protection system is similar to that for the DHW heating provided by the heat pumps. The inlet temperature $\left(55^{\circ} \mathrm{C}\right)$ and outlet temperature $\left(60^{\circ} \mathrm{C}\right)$ are both assumed to have uncertainties of $\pm 1^{\circ} \mathrm{C}$. The flow meter uncertainty is $\pm 5 \%$.

$$
\begin{gathered}
E_{\Delta T}=\sqrt{1^{2}+1^{2}}=1.4^{\circ} \mathrm{C} \\
e_{\Delta T}=\frac{1.4^{\circ} \mathrm{C}}{5^{\circ} \mathrm{C}}=0.28
\end{gathered}
$$

Adding the flow uncertainty in quadrature, the overall uncertainty for the DHW heating provided by the Legionella protection system is $\pm 28 \%$.

Calculation of uncertainty for the useful building heating provided (unintentionally) by the Legionella protection system in the form of heat leakage from piping was estimated as $\pm 3.3 \%$, based on the observed variation in the minimum electricity consumption. This does not include uncertainty due to the determination of whether or not the heat dissipated is useful. Presumably, the uncertainty regarding whether or not the heat dissipated is useful is higher during the shoulder seasons and lower during the peak heating and cooling seasons, but it seems unlikely to have a significant effect on the computed coefficients of performance for boundary H3.

\subsubsection{Uncertainty Analysis-Electrical Energy Consumed}

Although the electric meters are accurate to $\pm 1 \%$ and therefore add very little uncertainty to the coefficients of performance, our analysis requires several additional approximations that add additional uncertainty.

The additional uncertainty in the electrical energy associated with boundaries $\mathrm{H} 2$ and $\mathrm{C} 2$ is due to the electric meter measuring the heat pumps (including the circulating pumps that are inside the heat pump cabinets) and the Legionella protection system electrical energy, but not measuring the source-side circulating pump. Furthermore, the source-side circulating pump electrical energy must be allocated to heating and cooling. Then, the uncertainty in the electrical power and energy consumed by the heat pumps and source-side circulating pump under heating conditions for use in calculating $\mathrm{COP}_{\mathrm{H} 2}$ is given by:

$$
E_{P-H 2}=\sqrt{\left(e_{P} \cdot P\right)^{2}+f_{h t g} \cdot E_{S S C P}^{2}+E_{L P S}^{2}}
$$

where $e_{P}=$ fractional uncertainty in the meter measurement, $\pm 1 \% ; P=$ electrical energy measured by the meter, $\mathrm{kW} ; E_{S S C P}=$ absolute error in the estimate of the source-side circulating pump electrical energy consumption, $\pm 0.19 \mathrm{kWh} / \mathrm{h}$. 
For boundary condition C2, the only electricity consumption is that of the source-side circulating pump, so:

$$
E_{P-C 2}=f_{c l g} \cdot E_{S S C P}
$$

For boundary condition $\mathrm{H} 3$, the meter measurement includes the Legionella protection system, so the uncertainty is given by:

$$
E_{P-H 3}=\sqrt{\left(e_{P} \cdot P\right)^{2}+f_{h t g} \cdot E_{S S C P}^{2}}
$$

\subsubsection{Uncertainty Analysis-Coefficients of Performance}

With the uncertainties in heating/cooling provided and uncertainties in electrical energy determined, the uncertainties of the coefficients of performance can readily be determined. As shown by Taylor [65] if the uncertainties in the numerator and denominator are independent, the uncertainty of the quotient is given by:

$$
e_{q t}=\sqrt{e_{n}^{2}+e_{d}^{2}}
$$

where $e_{q t}, e_{n}, e_{d}$ are the fractional uncertainties of the quotient, numerator, and denominator respectively. This formulation was used to calculate the uncertainties for each COP boundary.

\section{Results}

This paper uses measured performance data from Studenthuset to characterize the actual thermal performance of the GSHP system during its third year of operation. Measured data from the period 1 April 2016 through 31 March 2017 are used. Monthly energy loads for this period are shown in Figure 3. This period was chosen because some additional instrumentation was added at the beginning of the period, allowing more accurate measurement of the cooling provided. The measured data, processed data, and Visual Basic for Applications computer code are available in the Supplementary Materials.

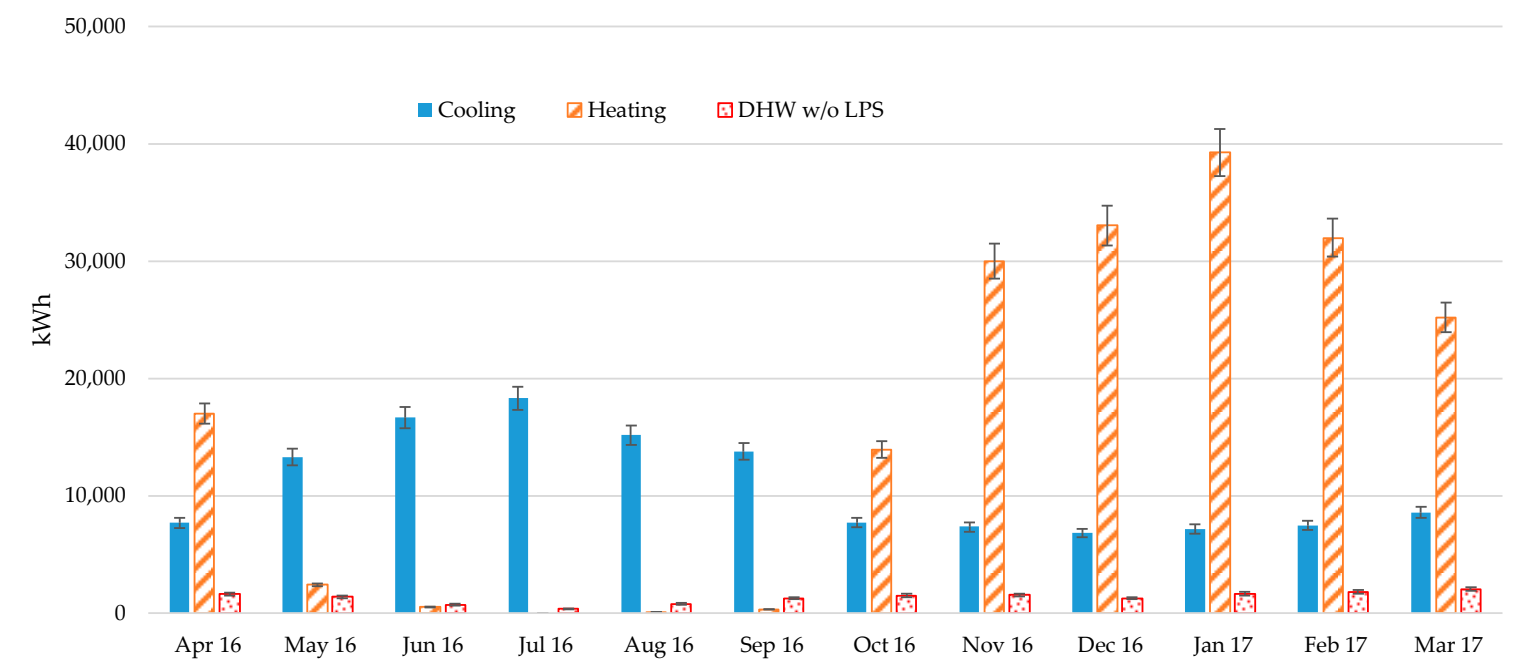

Figure 3. Monthly heating, cooling, and domestic hot water (DHW) load (not including Legionella protection energy) with uncertainty bars. 1 April 2016-31 March 2017.

\subsection{Load Characteristics}

The total energy loads for the selected period were 194 MWh space heating, 130 MWh space cooling, and 33 MWh domestic hot water (DHW) including the Legionella protection system (LPS).

As can be inferred from Figure 3, heating and cooling is provided simultaneously over a substantial part of the year. As discussed in Section 3.1.2, a complicating problem in determining the performance factors of the system is that there is no standard way to allocate electrical energy usage to cooling and 
heating respectively, when the system is doing both simultaneously. Equation (5) defined the "heating fraction" used to allocate the electrical energy consumption of the source-side circulation pump when the Studenthuset system is simultaneously providing building heating and DHW from the heat pumps while also providing cooling somewhere in the building. Figure 4 shows the heating fraction as a function of outdoor air temperature. Each point on the plot represents the average heating fractions for a particular outdoor air temperature bin of width $1^{\circ} \mathrm{C}$.

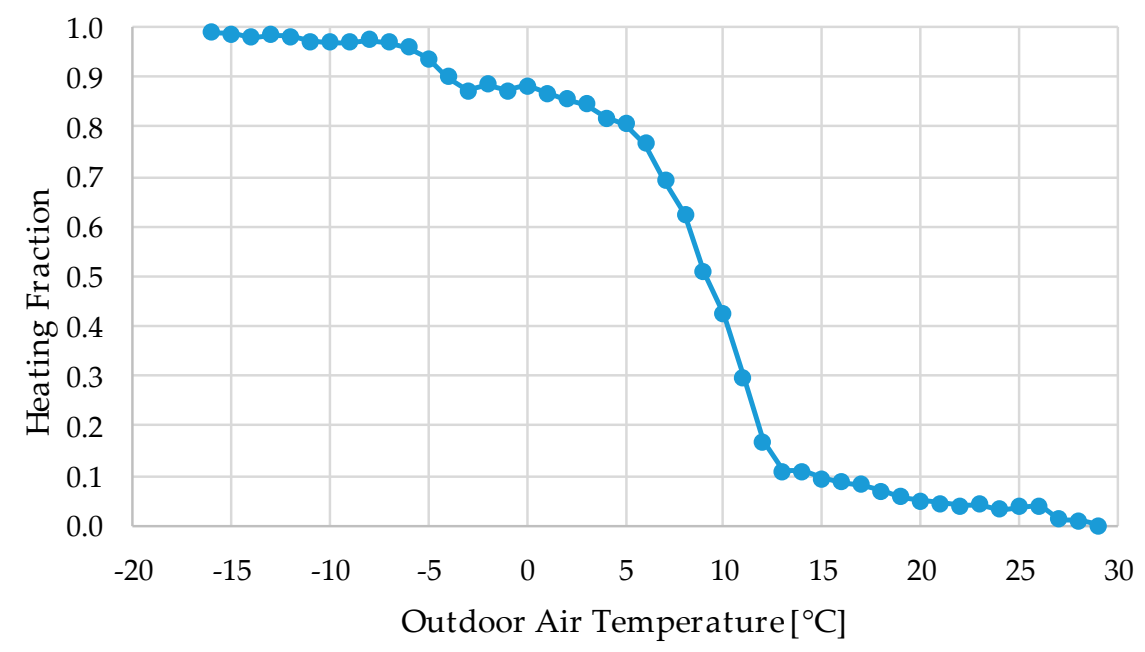

Figure 4. Heating fraction.

The heating system has a maximum capacity of $200 \mathrm{~kW}$, but as seen in the duration curve in Figure 5, heating is mostly provided at 20-60 kW. Maximum capacity of the borehole free-cooling system is $120 \mathrm{~kW}$.

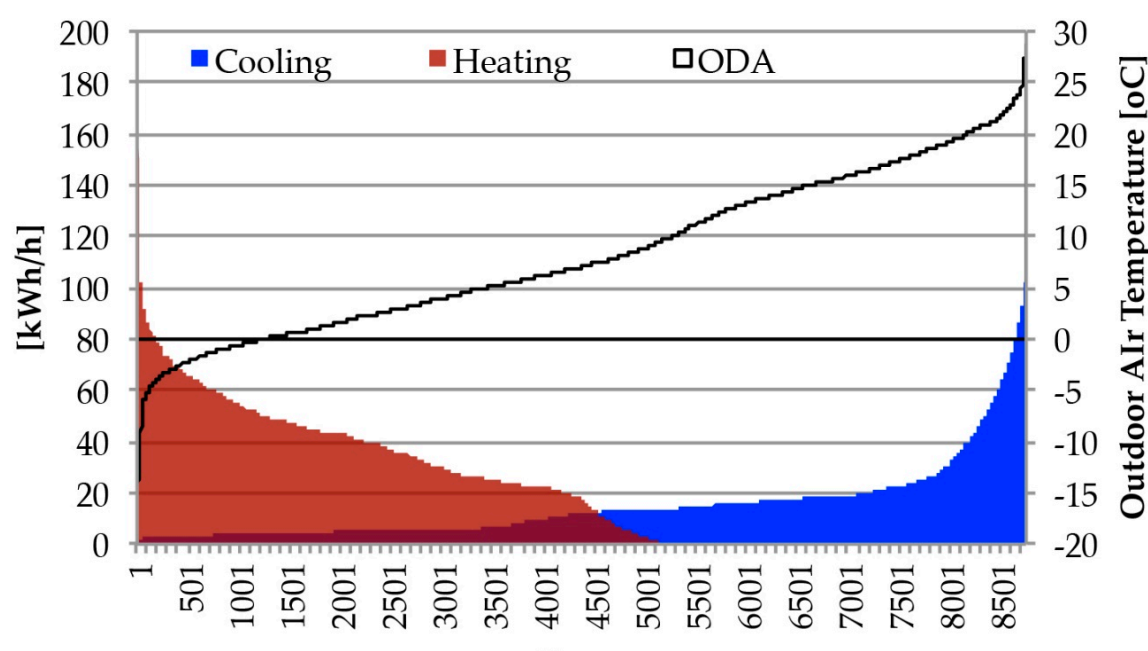

Hours

Figure 5. Duration curve for heating, cooling, and outdoor temperature (ODA). 1 April 2016-31 March 2017.

\subsection{System Boundaries}

In this paper we have used the system boundaries for calculating system performance for heating and cooling, as suggested by the EU project SEPEMO [22]. The SEPEMO project was mainly focused on single-family houses with heat pumps for heating or cooling, while Studenthuset is an office building with both heating and cooling, and thus has a higher degree of system complexity. Figure 6 shows the SEPEMO system boundaries applied to Studenthuset. 


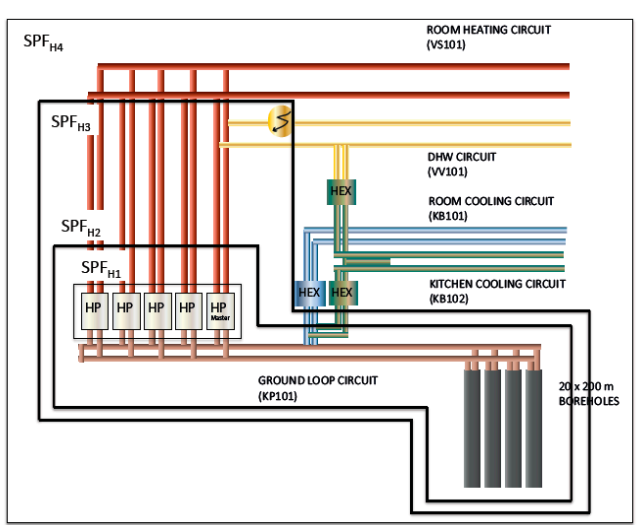

(a)

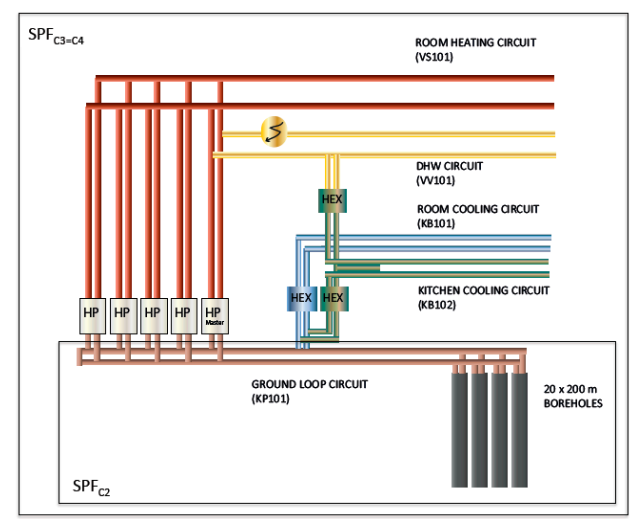

(b)

Figure 6. SEPEMO system boundaries [22] for heating SPF (a) and cooling SPF (b) for Studenthuset. $\mathrm{SPF}_{\mathrm{C} 1}$ cannot be calculated, as the system has no cooling unit.

Tables 3 and 4 summarize the energy delivered, and the allocated electricity usage for calculation of the various SPFs for heating and cooling respectively as defined by the SEPEMO system boundaries. $\mathrm{SPF}_{\mathrm{H} 1}$ cannot be calculated for Studenthuset as there is no instrumentation to measure heat pump compressor electricity separately from the built-in circulation pumps.

Table 3. Studenthuset heating SPF with SEPEMO system boundaries.

\begin{tabular}{|c|c|c|c|c|c|}
\hline Component & Annual (kWh) & $\mathrm{SPF}_{\mathrm{H} 1}$ & $\mathrm{SPF}_{\mathrm{H} 2}$ & $\mathrm{SPF}_{\mathrm{H} 3}$ & $\mathrm{SPF}_{\mathrm{H} 4}$ \\
\hline Delivered space heating from HP & $193,832 \pm 9785$ & $x$ & $x$ & $\mathrm{x}$ & $\mathrm{x}$ \\
\hline Delivered DHW heating from HP & $16,280 \pm 1260$ & $\mathrm{x}$ & $\mathrm{x}$ & $\mathrm{x}$ & $\mathrm{x}$ \\
\hline Delivered DHW heating from LPS & $1784 \pm 521$ & - & - & $\mathrm{x}$ & $\mathrm{x}$ \\
\hline Useful heat dissipated from LPS & $15,798 * \pm 598$ & - & - & $\mathrm{x}$ & $\mathrm{x}$ \\
\hline Useful heat dissipated - int. pumps and fans & $65,962 * \pm 660$ & - & - & - & $\mathrm{x}$ \\
\hline Delivered heating to building from EAHR & N/A & - & - & $* *$ & $\mathrm{x}$ \\
\hline Electricity HP compressor w/o int. circ. pump & N/A & $\mathrm{x}$ & - & - & - \\
\hline Electricity HP compressor + int. circ. pump & $52,208 \pm 1571$ & - & $\mathrm{x}$ & $\mathrm{x}$ & $\mathrm{x}$ \\
\hline Electricity source-side circ. pump for heating & $3998 * \pm 784$ & - & $\mathrm{x}$ & $\mathrm{x}$ & $\mathrm{x}$ \\
\hline Electricity LPS & $28,064 \pm 926$ & - & - & $\mathrm{x}$ & $x$ \\
\hline Electricity load-side circ. pumps + fans & N/A & - & - & - & $\mathrm{x}$ \\
\hline Electricity load-side circ. pumps + fans + EAHR & $48,293 * \pm 483$ & - & - & - & $\mathrm{x}$ \\
\hline SPF & $\mathrm{N} / \mathrm{A}$ & $4.0^{* * *} \pm 0.2$ & $3.7 \pm 0.2$ & $2.7 \pm 0.13$ & $\mathrm{~N} / \mathrm{A}$ \\
\hline
\end{tabular}

Table 4. Studenthuset cooling SPF with SEPEMO system boundaries.

\begin{tabular}{cccccc}
\hline Component Included & Annual (kWh) & SPF $_{\mathbf{C 1}}$ & SPF $_{\mathbf{C}}$ & SPF $_{\mathbf{C}}$ & SPF $_{\mathbf{C 4}}$ \\
\hline Delivered space cooling & $130,137 \pm 7054$ & $\mathrm{x}$ & $\mathrm{x}$ & $\mathrm{x}$ & $\mathrm{x}$ \\
\hline Delivered space cooling-supplemental cooling & 0 & - & - & - & $\mathrm{x}$ \\
\hline Delivered space cooling from ventilation & $\mathrm{N} / \mathrm{A}$ & - & - & $\mathrm{x}$ & $\mathrm{x}$ \\
\hline Electricity-cooling unit compressor & 0 & $\mathrm{x}$ & $\mathrm{x}$ & $\mathrm{x}$ & $\mathrm{x}$ \\
\hline Electricity-source-side circ. Pump-cooling & $4877^{*} \pm 880$ & - & $\mathrm{x}$ & $\mathrm{x}$ & $\mathrm{x}$ \\
\hline Electricity-load-side circ. pumps + fans & $57,148^{*} \pm 571$ & - & - & $\mathrm{x}$ & $\mathrm{x}$ \\
\hline Electricity suppl. cooling units & 0 & - & - & - & $\mathrm{x}$ \\
\hline SPF & N/A & N/A & $27 \pm 5$ & N/A & N/A
\end{tabular}

x: included; *: allocated; N/A: not applicable 
Part of the energy used by the Legionella protection system will be transferred to the building as heat. We treated this as "useful heat" for every hour for which the heat pumps were delivering heat to the building. In hours when no heating was otherwise being provided, it was assumed to be not useful and was not counted as delivered heating. In Tables 3 and 4 an asterisk $\left(^{*}\right)$ added to the annual $\mathrm{kWh}$ denotes that the number has been estimated by allocating between heating and cooling and, for components that start with "useful heat dissipated", the heating is only considered useful if there is space heating being delivered by the heat pumps during the hour. The uncertainty due to the determination of usefulness is not included in the uncertainty estimate. "N/A" means that the data is not available. Exhaust air heat recovery (EAHR) contributes to the heat delivered on the load-side, though it cannot be quantified with the existing instrumentation. Double asterisks $\left({ }^{* *}\right)$ in Table 3 indicate that the SEPEMO guidelines don't clearly speak to the question of whether or not heating provided by the EAHR system should be considered "auxiliary heating". The triple asterisks (***) in Table 3 for $\mathrm{SPF}_{\mathrm{H} 1}$ indicate that the calculated value does not conform exactly to the SEPEMO H1 boundaries, since the electrical energy includes all energy used by the heat pump units-including internal circulating pumps and control boards. Presumably, the actual $\mathrm{SPF}_{\mathrm{H} 1}$ should be somewhat higher.

As the Studenthuset cooling system does not include a cooling unit, there is no $\mathrm{SPF}_{\mathrm{C} 1}$. Also, there is no supplementary cooling for Studenthuset, hence $\mathrm{SPF}_{\mathrm{C} 3}=\mathrm{SPF}_{\mathrm{C} 4}$.

\subsection{Annual Performance}

Annual delivered space heating, space cooling, and DHW heating from the GSHP system is shown in Figure 7. Heat recovered from the ventilation air rotary heat exchangers is not included in the figure, as this energy could not be estimated from available data. Nor is the amount of cooling provided directly from the ventilation system.

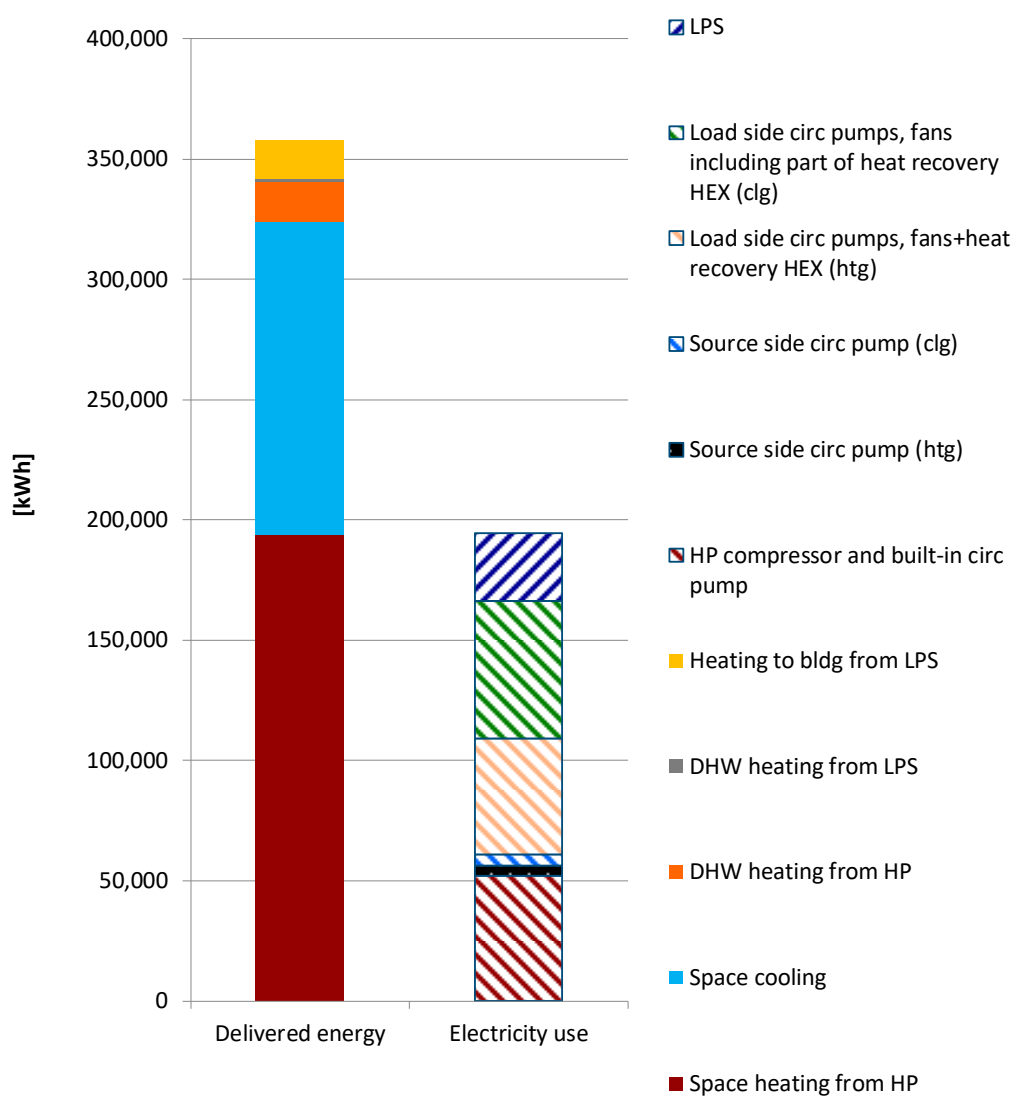

Figure 7. Annual delivered heating, cooling, and DHW provided (left), and electricity used (right). Note that delivered heating from exhaust air heat recovery is not included. 
Annual electricity used on the source-side and load-side is shown in the right hand column in Figure 7. This electricity consumption used for heat recovery is lumped in with all of the distribution pump and fan energy, and cannot readily be quantified separately. Without accounting for the recovered heat in the ventilation air or the "free" cooling sometimes provided by the ventilation air, $\mathrm{SPF}_{\mathrm{H} 4}, \mathrm{SPF}_{\mathrm{C} 3}$, and $\mathrm{SPF}_{\mathrm{C} 4}$ cannot be fairly calculated. Figure 7 shows that electricity use for distribution of heating and cooling, ventilation, and heat recovery accounts for about twice as much electricity as the heat pumps and source-side circulating pump together. In a centralized system such as this, distribution energy on the load-side may be similar regardless of the chosen energy source. That is, the distribution energy may be approximately the same whether the heating is provided by centralized GSHPs, district heating, or a gas boiler. Therefore, SPF and COP for H3 and C2 boundaries may still be useful for comparison to other heating and cooling system types. However, for comparison to distributed GSHP systems, it is desirable to make comparisons using the $\mathrm{H} 4$ or $\mathrm{C} 3$ boundaries, because the distributed GSHP energy necessarily includes fan power.

\subsubsection{Heating}

Delivered space heating, electricity consumption for heating, and seasonal performance factors for the heating system are shown in Table 3. As discussed above, the electricity used by the source-side circulation pump had to be allocated between heating and cooling based on the heating fraction. The total annual space heating delivered to the building, excluding heat recovery, was $194 \mathrm{MWh}$ which is consistent with the anticipated annual space heat load (200 MWh) for the building.

According to the building design documents, the SPF for the heat pumps was expected to be 4.5. As mentioned above, the design documents are silent on the point of which boundary conditions correspond to the stated SPF. Annual heating SPF for the heat pumps including electricity for the internal circulation pumps and control boards was $4.0 \pm 0.2$. As noted above, this value should be a little lower than $\mathrm{SPF}_{\mathrm{H} 1}$, because of the additional electrical energy being included. Annual $\mathrm{SPF}_{\mathrm{H} 2}$ (which includes both the internal and external circulation pumps) was $3.7 \pm 0.2$. Taking into account the supplemental heating provided by the Legionella protection system, $\mathrm{SPF}_{\mathrm{H} 3}$ is $2.7 \pm 0.13$. The lower value is due to the supplemental heating being provided by an electric resistance heater and a recirculation pump.

Taking the annual heating SPF for the heat pumps including electricity for the internal circulation pumps as a surrogate for $\mathrm{SPF}_{\mathrm{H} 1}$, we can see that $\mathrm{SPF}_{\mathrm{H} 1}$ and $\mathrm{SPF}_{\mathrm{H} 2}$ are $18 \%$ and $10 \%$ lower, respectively, than the median values reported in the literature review (Table 2). The 18 and 16 cases for which $\mathrm{SPF}_{\mathrm{H} 1}$ and $\mathrm{SPF}_{\mathrm{H} 2}$ are reported are all for buildings situated in warmer climates with warmer ground temperatures, which may explain part of the reason for this. Other reasons for sub-optimal performance are discussed below.

\subsubsection{Cooling}

Seasonal performance factors for the cooling system are shown in Table 4. Studenthuset does not have a cooling unit, hence $\mathrm{SPF}_{\mathrm{C} 1}$ does not exist, but $\mathrm{SPF}_{\mathrm{C} 2}$ which includes the source and source-side circulation pump is estimated as $27 \pm 5$. Electricity used by source-side circulation pumps for cooling is allocated using the cooling fraction defined by Equation (6). As the amount of cooling provided by the ventilation system is not measured, $\mathrm{SPF}_{\mathrm{C} 3}$ and $\mathrm{SPF}_{\mathrm{C} 4}$ cannot be estimated.

Measured annual cooling amounts to approximately $130 \mathrm{MWh}$, which is about four times as much as the anticipated annual design cooling-load of $34 \mathrm{MWh}$. The reason for this difference has not yet been determined.

Compared to the nine cases for which $\mathrm{SPF}_{\mathrm{C} 2}$ is reported in the literature, the Studenthuset system has significantly higher $\mathrm{SPF}_{\mathrm{C} 2}$, about 3.4 times the median value. This is primarily due to direct use of cold ground heat exchanger fluid. It may also be partly explained that the system is always providing some heating, so some of the pumping energy is always allocated to heating, thus reducing the energy consumed for cooling. 


\subsection{Monthly Performance}

\subsubsection{Heating}

Monthly performance factors for heating were calculated using the system boundaries in Figure 6. Figure 8 shows monthly $\mathrm{COP}_{\mathrm{H} 2}$ and $\mathrm{COP}_{\mathrm{H} 3}$ for the evaluated period.

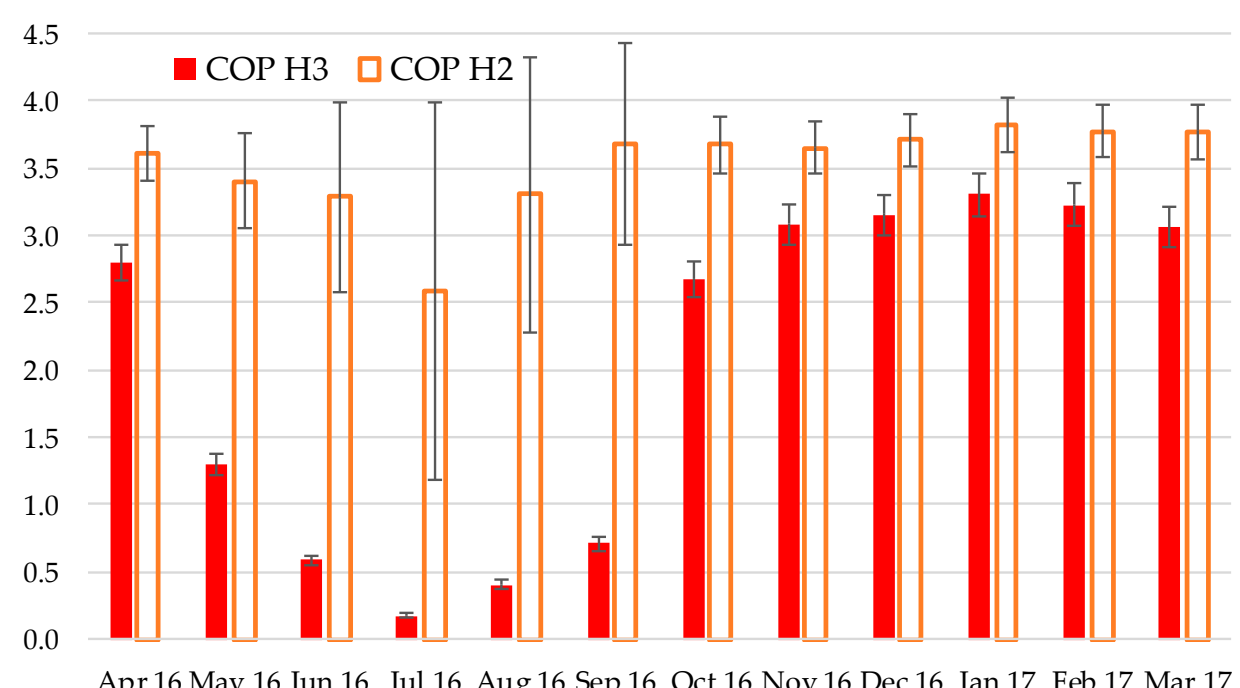

(a)

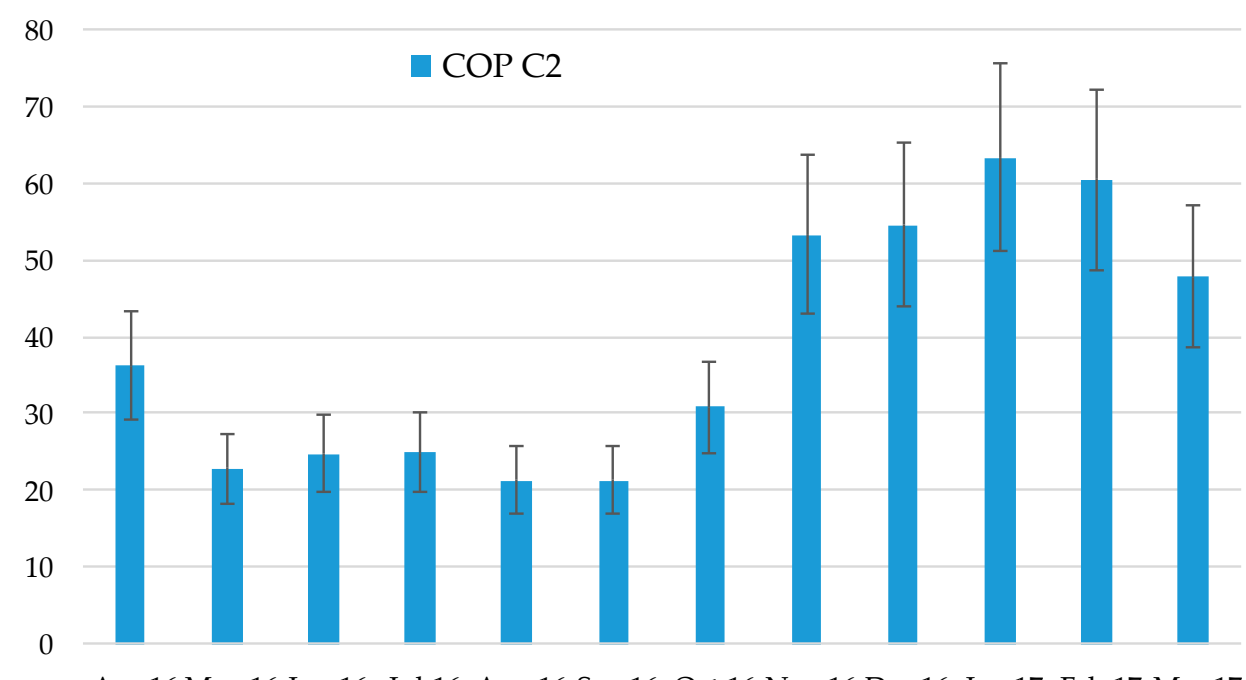

Apr 16 May 16 Jun 16 Jul 16 Aug 16 Sep 16 Oct 16 Nov 16 Dec 16 Jan 17 Feb 17 Mar 17

(b)

Figure 8. Monthly $\mathrm{COP}_{\mathrm{H} 2}$ and $\mathrm{COP}_{\mathrm{H} 3}(\mathbf{a})$ and $\mathrm{COP}_{\mathrm{C} 2}$ (b) with uncertainty bars, 1 April 2016-31 March 2017.

Somewhat counter-intuitively, the monthly heating COPs are highest during the colder months and lowest in the summer. The explanation lies partly in the source-side circulation pumps providing a minimum of $8 \mathrm{~L} / \mathrm{s}$ throughout the year, regardless of whether or not there is a heating load. $\mathrm{COP}_{\mathrm{H} 3}$ is largely affected by the Legionella protection system (LPS), which runs all the time. Much of the electricity consumed by the LPS is eventually dissipated to the building, so during the heating season, this is counted as "useful heating delivered", increasing $\mathrm{COP}_{\mathrm{H} 3}$. However, during the warmer months, the heat dissipated is not useful and it significantly decreases $\mathrm{COP}_{\mathrm{H} 3}$ to the point that it falls below 1 for some months. 
The uncertainty for boundary $\mathrm{H} 3$ is generally lower than that for $\mathrm{H} 2$. This is largely due to the fact that the electrical energy can be measured more accurately for boundary $\mathrm{H} 3$ than for $\mathrm{H} 2$.

\subsubsection{Cooling}

Monthly cooling coefficients of performance for boundary C2 are presented in Figure 8 . The COP varies between around 20 in the summer to above 60 during the winter, when the heat pumps are lowering the heat carrier fluid temperature. The amount of cooling used in the winter months is however quite small. These COPs do not account for distribution energy (as in $\mathrm{COP}_{\mathrm{C} 3}$ and $\mathrm{COP}_{\mathrm{C} 4}$ ). $\mathrm{COP}_{\mathrm{C} 3}$ cannot currently be calculated due to lack of data from the ventilation system. As $\mathrm{COP}_{\mathrm{C} 3}$ takes into account cooling provided by the ventilation system and the energy of the pumps and fans, it is difficult to speculate as to how values of $\mathrm{COP}_{\mathrm{C} 3}$ will compare to $\mathrm{COP}_{\mathrm{C} 2}$.

\subsection{Performance vs. Temperature and Load}

In Figure $9 \mathrm{COP} \mathrm{H} 3, \mathrm{H} 2$, and $\mathrm{C} 2$, binned at half-degree intervals, are plotted versus entering fluid temperature (EFT) to the system from the borehole field. By "binned" we mean that average values are taken for all hours with heat pump EFT that fall within a half-degree bin, e.g., the points shown at $10{ }^{\circ} \mathrm{C}$ represent the total heating provided divided by the electricity used for all hours with heat pump EFT between $9.75^{\circ} \mathrm{C}$ and $10.25^{\circ} \mathrm{C}$.

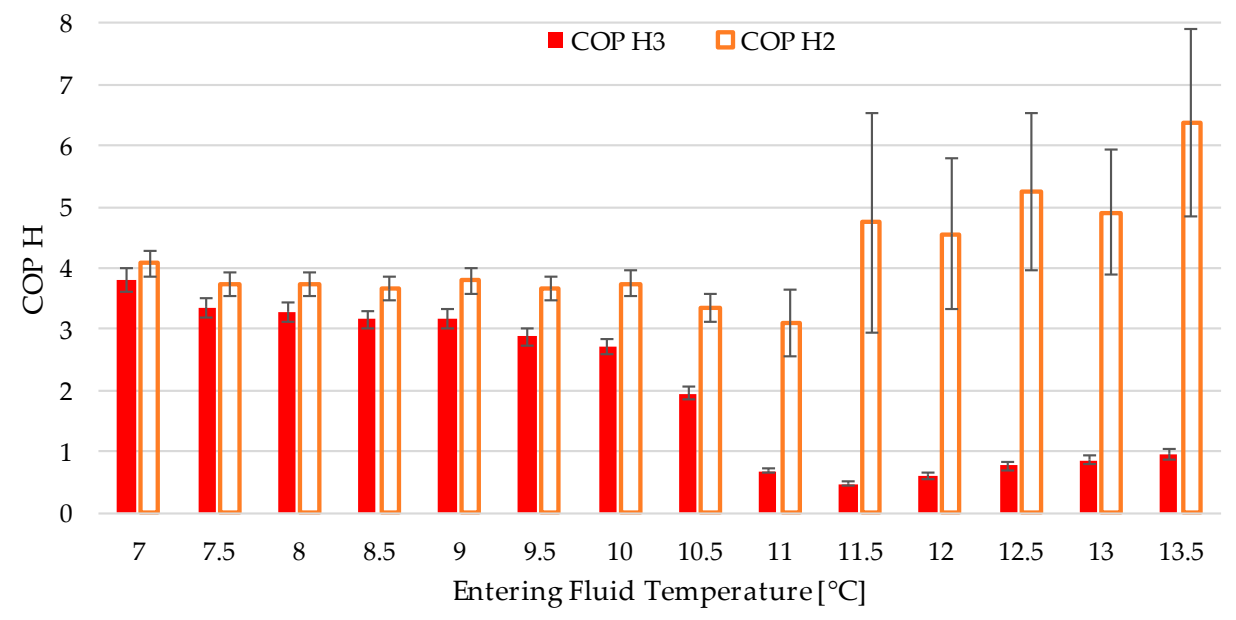

(a)

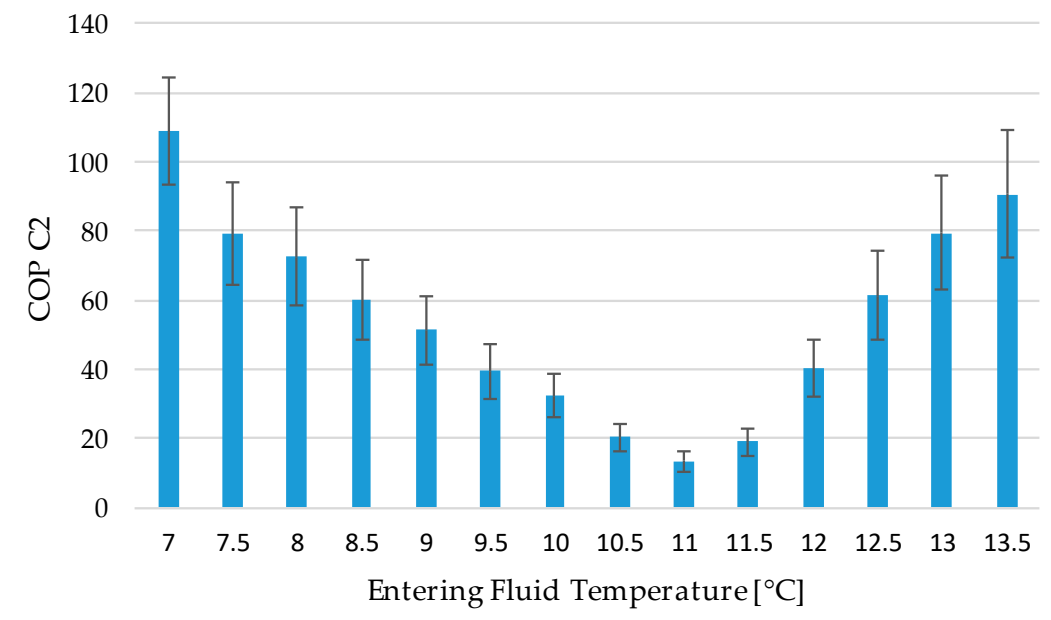

(b)

Figure 9. $\mathrm{COP}_{\mathrm{H} 2}$ and $\mathrm{COP}_{\mathrm{H} 3}(\mathbf{a})$ and $\mathrm{COP}_{\mathrm{C} 2}(\mathbf{b})$ vs. entering fluid temperature (EFT) with uncertainty bars. 
$\mathrm{COP}_{\mathrm{C} 2}$ and $\mathrm{COP}_{\mathrm{H} 2}$ have minimums at $11^{\circ} \mathrm{C}$ and $\mathrm{COP}_{\mathrm{H} 3}$ drops significantly between $10-11.5^{\circ} \mathrm{C}$. As shown in Figure 10 the overall load is as lowest at those EFTs. This correlation between low part loads and low system performance is consistent with measured performance of the distributed GSHP system [51] serving an office building in Atlanta and also the central GSHP system [60] serving a university building in Leicester, England. This may seem counterintuitive, compared to thermodynamic theory, which might lead us to expect that the highest heating COP should occur at the highest heat pump EFT. But the effects of "parasitic" loads—e.g., pumping and control boards—and heat pump cycling losses seem to dominate the actual field-measured COP. For $\mathrm{COP}_{\mathrm{H} 3}$, the very low values at temperatures above $10.5^{\circ} \mathrm{C}$ are largely due to the Legionella protection system, as discussed in the previous section regarding performance during summer months.

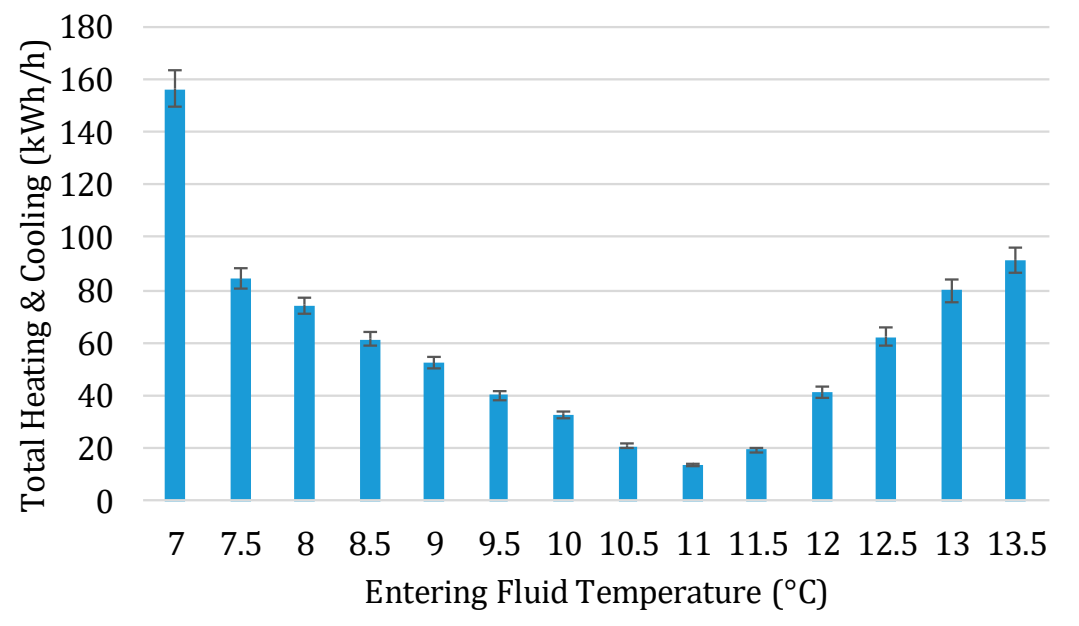

Figure 10. Overall load vs. EFT.

Figure 11 shows daily overall COP plotted versus overall energy load. Loads include building heating and DHW heated by the heat pumps and the Legionella protection system (LPS), while for cooling only the chilled water provided by the boreholes is included. The electricity use includes heat pump operation, LPS, and source-side circulating pump. Days with mainly cooling load (heating fraction $\leq 0.25$ ), mainly heating load (heating fraction $\geq 0.67$ ), and mixed heating and cooling load are color coded to illustrate the different correlations between COP and heating and cooling load respectively.

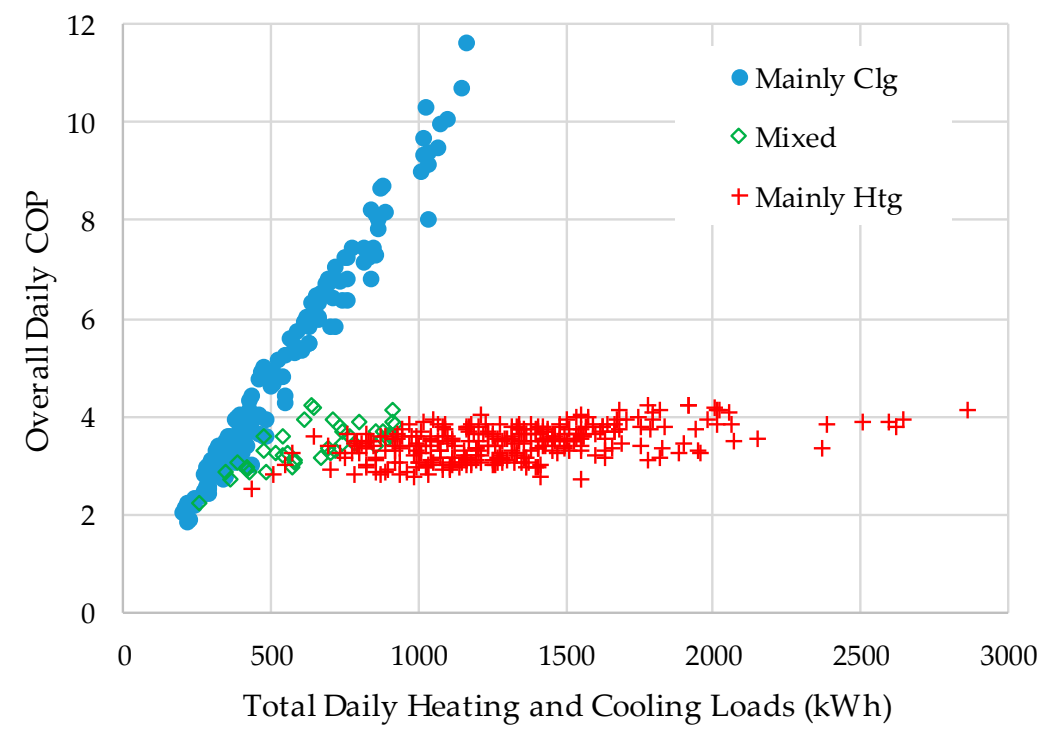

Figure 11. Overall daily COP vs. overall daily load. 
The figure raises some interesting questions. Firstly, why is there such a discrepancy between $\mathrm{COP}_{\mathrm{C} 2}$ in Figures 8 and 9, and the overall COP for the mainly cooling cases? The explanation is that for days when the heating fraction is very small, there is still a DHW load, and the LPS is still running. For an outdoor air bin temperature of $20^{\circ} \mathrm{C}$, the cooling provided is about $40 \mathrm{~kW}$ and the DHW provided is about $2.3 \mathrm{~kW}$, but the LPS is using $3.2 \mathrm{~kW}$ and the circulating pump is about $1 \mathrm{~kW}$. This means that even though $\mathrm{COP}_{\mathrm{C} 2}$ at those conditions is 42 , adding the DHW heating gives about $42.3 / 4.2 \mathrm{~kW}$, which gives an overall COP of only 10.1.

Second, we don't see nearly as strong a trend for the heating loads as for the cooling loads. COPs for cooling dominated conditions increase about 10 units over $1000 \mathrm{kWh}$ increased daily energy load, whereas the increase in heating dominated COPs is only 1 unit over $2500 \mathrm{kWh}$ load increase. While an increased cooling load only requires a small increase in electricity use for the circulation pump to increase the flow rate, it also means that the relative influence on the COP from the Legionella protection system and DHW circulation decreases. For heating dominated conditions, the Legionella protection system and DHW circulation are small contributions to the overall load and electricity use. Also, since there are five heat pumps that are staged, individual heat pumps tend to work at close to full load conditions.

\section{Discussion}

System performance has been analyzed based on boundary conditions $\mathrm{H} 2, \mathrm{H} 3$, and $\mathrm{C} 2$ shown in Tables 3 and 4 . The results have been presented for a one-year period, monthly periods, daily periods, hourly periods, and binned hourly periods.

Uncertainties in heating/cooling provided, COPs, and SPFs are provided. Because uncertainties for performance parameters based on field measurements have rarely been presented in the literature, there is little to compare these values to, with the exception of Southard et al. [49], Urchueguía et al. [37], and Hughes [41]. The uncertainties in the present study are similar in magnitude or smaller to those presented in either Southard et al. [49], Urchueguía et al. [37], or Hughes [41]. The present study has the advantage of using matched pairs of temperature sensors that significantly reduce the uncertainty in the temperature difference measurement. However, the Southard et al. [49] study had the advantage of making separate measurements on 14 different heat pumps; aggregation of the different measurements reduces the uncertainty of the total heating or cooling provided by all heat pumps. It would be desirable to check the accuracy of the individual sensors and sensor pairs after several years of operation in order to insure that the sensors have not drifted from their calibration.

For the situation in Sweden, the annual SPFs $\mathrm{SPF}_{\mathrm{H} 2}$ or $\mathrm{SPF}_{\mathrm{H} 3}$ are commonly used as a basis for comparison, because other systems to which GSHP systems may be compared are also centralized systems. That is, they also provide hot water and chilled water to panel radiators, fan coil units, and other heating/cooling distribution devices. In this case, it makes sense to compare the "central plant" equipment using the $\mathrm{H} 2$ and $\mathrm{H} 3$ boundary conditions. For cooling, the $\mathrm{C} 2$ boundary conditions would be commonly used.

However, if we wish to compare to distributed GSHP systems or other distributed systems where the heating and cooling equipment has integrated distribution fans and, likely, ventilation provided using these same fans, it is necessary to compare using boundary conditions that include the heating and cooling distribution and even the ventilation. In our results for the Studenthuset system, these would correspond to the $\mathrm{H} 4$ and $\mathrm{C} 4$ results.

This, however, raises a further complication. The ventilation system does provide heating and cooling because outdoor air is at least tempered using hot water or chilled water from the heat pumps. Therefore, the ventilation system electricity consumption is included in the H4 and C4 boundary conditions. On the other hand, the ventilation system consumes electricity primarily for the purpose of providing fresh air to the space, not for heating and cooling purposes. So, when calculating heating and cooling COPs with the $\mathrm{H} 4$ and $\mathrm{C} 4$ boundary conditions, is there a fair way to divide the electricity consumption between heating/cooling and provision of fresh air? This has not been addressed in 
the literature. It seems that it might be possible to calculate the pressure drop due to heating and cooling coils, the pressure drops due to the rest of the ventilation system, and apportion the electricity consumption accordingly. This remains a topic for further investigation.

Beyond the question of the comingling of electricity for ventilation and heating/cooling, several other problematic issues have arisen during the performance analysis of the Studenthuset system. These include:

- Division of electricity consumption between heating and cooling when both are being provided simultaneously. For the Studenthuset building, this arises when accounting for the electricity used by the source-side circulating pump under conditions when one or more heat pumps are being operated to provide heating and "free" cooling is being provided at the same time directly from the boreholes. It also arises with respect to the fans and pumps of the heating/cooling distribution system and ventilation system, for which we only have a single energy meter. In these cases, we divided the electricity proportionally to the amount of heating and cooling provided.

- The Studenthuset system utilizes an electric resistance heater to raise the domestic hot water temperature in order to protect against Legionella. The required hot water temperature is $60{ }^{\circ} \mathrm{C}$, but in practice the electric resistance heater is operated continuously and excess heat is dissipated into the building. A pump is used to continuously circulate this hot water around the building. This continuous circulation is used both to protect against Legionella (by insuring that temperatures remain high throughout the domestic hot water piping) and to significantly reduce the amount of waiting time for hot water at any tap. Treatment of this subsystem is perplexing. Should the heating provided by the electric resistance heater all be treated as providing useful heating to the building? Clearly, when domestic hot water is being utilized, the Legionella protection system is providing some of the required heating. The energy expended to heat the domestic hot water to $60^{\circ} \mathrm{C}$ can certainly be considered as part of the heating load. But what about that dissipated to the building? It could either be providing useful heating or merely increasing the cooling load. So, it seems that it should be treated on the basis as to whether or not the building has a heating load at that time. In this analysis, we took this into account by treating the heat provided by the Legionella protection system as useful for any hours where the heat pumps were providing any building heating. However, as this includes hours when the building was simultaneously being heated and cooled, this approach should be refined.

- The fact that the Legionella protection system ran every single hour did, however, alleviate one other problem encountered in Southard et al. [49]—how to treat energy consumed by pumps, fans, control boards, etc., when neither heating nor cooling are provided by the system. Hughes [41] also identifies Legionella protection systems as problematic for the overall system performance, as they significantly increase the use of auxiliary heating with immersion heaters. He observes that several of the GSHP systems in his study do not appear to be operating according to current best practice.

- It may always be the case that additional instrumentation is desirable. In this case, we could not calculate $\mathrm{COP}_{\mathrm{H} 1}$ because the heat pumps had integrated circulating pumps and we did not have independent measurements of the compressor energy consumption.

- Another limitation in the instrumentation is that heating supplied and cooling supplied are determined solely from water-side measurements made near the central plant. As shown in Figure 7, the internal circulation pumps and fans use a little over half of all system electricity. This electricity usage would be accounted for in the input electricity used to calculate COP and SPF for boundaries H4, C3, and C4. However, all of this electricity is eventually dissipated as heat somewhere. Treatment of this dissipated heat faces the same complications as the treatment of heat from the Legionella protection system. Furthermore, the fan energy is used to provide heating and cooling to the ventilation air and cooling to the building space. But without suitable instrumentation, it is not possible to quantify this. Therefore, we demurred from 
calculating COP and SPF for boundaries $\mathrm{H} 4, \mathrm{C} 3$, and C4. Further instrumentation here would be particularly helpful.

Finally, one of the most important uses of this kind of performance investigation is identification of possible system improvements. We have identified several possibilities, though their impacts have not yet been fully evaluated:

- The scheduled run time of the electric resistance heater providing Legionella protection could be reduced. It currently runs $24 \mathrm{~h}$ per day, seven days per week. Many heat pump systems run a heating cycle for Legionella protection on a once-per-week schedule. Wemhoener et al. [33] describe a Legionella protection system running twice per week. Hughes $[40,41]$ describes a Legionella protection method where the temperature at the top of the DHW storage tank is kept at $60{ }^{\circ} \mathrm{C}$, and an immersion heater is used to raise the temperature in the whole tank to $60{ }^{\circ} \mathrm{C}$ for a minimum of one hour per day. One of the GSHP systems reported by Hughes $[40,41]$ uses ultraviolet light for disinfection; however, that system already has a high DHW output temperature of above $62^{\circ} \mathrm{C}$.

- The continuous circulation pump could also be scheduled to run at a reduced run time, more closely matching the operating hours of the building. Merely limiting the circulation pump and electric resistance heating to run during office hours only would save about 12,000 kWh/year in electricity consumption. Some of the heating provided by the Legionella protection system would then be replaced with heat provided by heat pumps at a higher COP.

- The minimum flow rate through the ground heat exchanger is set to be $8 \mathrm{~L} / \mathrm{s}$ year-round. This often results in very low $\Delta \mathrm{T}$ across the boreholes. It seems likely that the setpoint could be reduced significantly for portions of the year and/or a reset control could be utilized that reduces the flow when allowable.

\section{Conclusions}

The Studenthuset in Stockholm, Sweden, and its GSHP system were designed with high ambitions for both energy efficiency and indoor comfort. The owner has long experience with GSHP systems in buildings and the system is thoroughly instrumented and monitored by experienced staff. One notable gap in the instrumentation (airflow rate measurement) precludes calculating COP and SPF for the entire system. This study shows that the system provides space heating consistent with the design values, and that the cooling provided is about four times higher than anticipated from the design.

An SPF for SEPEMO boundary H2 (heat pump + source-side circulating pump) of $3.7 \pm 0.2$ was achieved. An SPF for boundary H3 (heat pump + source-side circulating pump + auxiliary heating) of $2.7 \pm 0.13$ was achieved. The $\mathrm{SPF}_{\mathrm{H} 2}$ value is lower than most of the reported values in the literature, which have a median value of 3.6. The $\mathrm{SPF}_{\mathrm{H} 3}$ value is lower than the only reported value of $\mathrm{SPF}_{\mathrm{H} 3}$ in the literature. For cooling, the $\mathrm{SPF}_{\mathrm{C} 2}$ value of $27 \pm 5$ is considerably higher than any value reported in the literature. These values are partly explained by the relatively lower ground temperatures found in Stockholm.

However, a key finding is that the measured COPs are more affected by the amount of heating and cooling provided than by the entering fluid temperature to the heat pumps. As shown by the bin analysis, lower entering fluid temperatures correspond to higher run time fractions for equipment and less influence of "parasitic" loads such as pumps and unit control boards. This is consistent with findings for both a distributed GSHP system [51] and a central GSHP system [60]. For this system, the Legionella protection system and DHW recirculation system, which are always on, form a significant parasitic load. The near-constant flow rate in the borehole circuit leads to the circulating pump also acting as a significant parasitic load. Therefore, reduced scheduling of the Legionella protection and DHW recirculation systems and allowing reduced flow in the borehole circuit are recommended actions to significantly decrease the parasitic losses, improve the performance, and decrease the energy performance gap. 
This paper presents an approach to addressing the performance gap without installing research-grade instrumentation. High-quality commercially-available instrumentation selected by the owners for the building energy management system proved sufficient to measure the system SPFs and COPs for several of the SEPEMO boundary conditions with reasonable accuracy, as demonstrated with the uncertainty analysis. Another contribution of this paper is the use of a bin analysis of system COPs to identify several problems with the operating strategy that, once corrected, have significant potential to reduce the system energy consumption.

Despite the thorough instrumentation and monitoring, several additional measurement points are needed in order to provide a complete analysis of the system performance. Additional instrumentation and data logging would be needed for estimating the amount of heat recovery and cooling provided from the ventilation air, as well as allocating the electricity used for this. This would make possible estimation of $\mathrm{SPF}_{\mathrm{H} 4}$ and $\mathrm{SPF}_{\mathrm{C} 3}$ and $\mathrm{SPF}_{\mathrm{C} 4}$.

Other desirable additional instrumentation would be electricity measurements for the heat pump compressors to enable estimation of $\mathrm{SPF}_{\mathrm{H} 1}$, separate electricity measurement for the circulation pump on the source-side, and separate electricity measurements for the different parts of the Legionella protection system.

For a Swedish centralized GSHP system like Studenthuset, it makes sense to evaluate $\mathrm{SPF}_{\mathrm{H} 2 \text {, }}$ $\mathrm{SPF}_{\mathrm{H} 3}, \mathrm{SPF}_{\mathrm{C} 2}$, and an overall SPF including only the ground source, heat pumps, and DHW heating, and leaving out the load-side energy and electricity. However, since $\mathrm{SPF}_{\mathrm{H} 4}$ and $\mathrm{SPF}_{\mathrm{C} 3}\left(=\mathrm{SPF}_{\mathrm{C} 4}\right)$ could not be calculated for Studenthuset, it is not possible to compare this centralized system with a decentralized system such as the ASHRAE HQ in Atlanta.

Finally, this study has identified several issues in calculation of SPF and COP for commercial buildings that are not addressed in the literature or by existing boundary definition schema. For example, treatment of electricity used by ventilation fans, for which the main purpose is providing ventilation, but which also provide significant amounts of heating and cooling. Further research in this area is part of an ongoing collaborative research project [66] organized by the International Energy Agency Heat Pumping Technologies Collaboration Program.

Supplementary Materials: The following are available online at http://www.mdpi.com/1996-1073/12/10/2020/s1, Excel workbook containing hourly data, VBA source code used for analysis, and plots for Figures 3-5, Figures 7-11, and Tables 3 and 4: Studenthuset_GSHP_data_201604-201703.xlsm.

Author Contributions: Both authors contributed to the paper as follows. Conceptualization, J.D.S. and S.G.; methodology, J.D.S. and S.G.; software, J.D.S.; validation, J.D.S. and S.G.; formal analysis, J.D.S. and S.G.; investigation, J.D.S. and S.G.; resources, J.D.S. and S.G.; data curation, J.D.S. and S.G.; writing-original draft preparation, J.D.S. and S.G.; writing—review and editing, J.D.S. and S.G.; visualization, J.D.S. and S.G.; supervision, J.D.S. and S.G.; project administration, J.D.S. and S.G.; funding acquisition, S.G.

Funding: This research was partially funded by the Swedish Energy Agency through the TERMO research program, grant number 45979-1.

Acknowledgments: Thanks to Anders Larsson and Åke Annsberg at Akademiska Hus for their help and support, and providing measurement data and information about the monitored building. Special thanks to Akademiska Hus for granting permission to publically share the data. The support from the authors' employers is gratefully acknowledged. This work is part of the IEA HPT Annex 52, long term performance measurement of GSHP systems serving commercial, institutional, and multi-family buildings [66]. Interactions with our international colleagues have been helpful in influencing our thinking on addressing the performance gap with monitoring.

Conflicts of Interest: The authors declare no conflict of interest.

\section{Nomenclature}

$c_{p} \quad$ specific heat of the water, $\mathrm{kJ} /(\mathrm{kg} \cdot \mathrm{K})$

$\mathrm{COP}_{\mathrm{H} 1} \quad$ Coefficient of performance over a given period of time (e.g., a day or a month) for the system boundary heat pump only

$\mathrm{COP}_{\mathrm{H} 2} \quad$ Coefficient of performance over a given period of time (e.g., a day or a month) for the system boundary heat pump and circulation pumps/fans on source-side 
$\mathrm{COP}_{\mathrm{H} 3} \quad$ Coefficient of performance over a given period of time (e.g., a day or a month) for the system boundary heat pump, circulation pumps/fans on source-side and auxiliary heating

$\mathrm{COP}_{\mathrm{H} 4}$ Coefficient of performance over a given period of time (e.g., a day or a month) for the system boundary heat pump, circulation pumps/fans on source-side, auxiliary heating and circulation pumps/fans on load-side

$\mathrm{COP}_{\mathrm{C} 1} \quad$ Coefficient of performance over a given period of time (e.g., a day or a month) for the system boundary cooling unit only

$\mathrm{COP}_{\mathrm{C} 2} \quad$ Coefficient of performance over a given period of time (e.g., a day or a month) for the system boundary cooling unit and circulation pumps/fans on source-side

$\mathrm{COP}_{\mathrm{C} 3} \quad$ Coefficient of performance over a given period of time (e.g., a day or a month) for the system boundary cooling unit, circulation pumps/fans on source-side and load-side

$\mathrm{COP}_{\mathrm{C} 4}$ system boundary cooling unit, circulation pumps/fans on source-side and load-side, and supplementary cooling unit

fractional uncertainty of the flow rate

fractional uncertainty of the temperature $(\mathrm{K})$

fractional uncertainty of the denominator

fractional uncertainty of the numerator

fractional uncertainty of the quotient

fractional uncertainty in the meter measurement

fractional uncertainty of the heat transfer rate

absolute uncertainty of the temperature (K)

uncertainty in the electrical power and energy consumed by the heat pumps and source-side circulating pump under heating conditions for use in calculating $\mathrm{COP}_{\mathrm{H} 2}$ uncertainty in the electrical power and energy consumed by the heat pumps and source-side circulating pump under heating conditions for use in calculating $\mathrm{COP}_{\mathrm{H} 3}$ absolute uncertainty in building heating provided for a one-month period $(\mathrm{kWh})$ absolute error in the estimate of the source-side circulating pump electrical energy consumption, $\mathrm{kWh} / \mathrm{h}$

$f_{\text {clg }}$

$f_{h t g}$

n fraction of electrical energy consumed by the circulating pump that is allocated to the heating electrical energy fraction of electrical energy consumed by the circulating pump that is allocated to the heating electrical energy

day of the year

day of the year with minimum temperature (day 60)

electrical energy measured by the meter, $\mathrm{kW}$

electrical power $(\mathrm{kW})$ consumed as a function of flow rate average heating provided to the building over the time interval, $\mathrm{kW}$ average cooling provided to the building over the time interval, $\mathrm{kW}$ average heating provided to the domestic hot water over the time interval, $\mathrm{kW}$ average heating energy $(\mathrm{kWh})$ provided by the heat pumps to the domestic hot water energy provided by the electric resistance heater for the Legionella protection system Seasonal performance factor over 365 consecutive days for the system boundary H1 (heat pump only)

$\mathrm{SPF}_{\mathrm{H} 2} \quad$ Seasonal performance factor over 365 consecutive days for the system boundary H2 (heat pump and circulation pumps/fans on source-side)

$\mathrm{SPF}_{\mathrm{H} 3} \quad$ Seasonal performance factor over 365 consecutive days for the system boundary H3 (heat pump, circulation pumps/fans on source-side and auxiliary heating)

$\mathrm{SPF}_{\mathrm{H} 4} \quad$ Seasonal performance factor over 365 consecutive days for the system boundary H4 (heat pump, circulation pumps/fans on source-side, auxiliary heating, and circulation pumps/fans on load-side)

$\mathrm{SPF}_{\mathrm{C} 1} \quad$ Seasonal performance factor over 365 consecutive days for the system boundary $\mathrm{C} 1$ (cooling unit only)

$\mathrm{SPF}_{\mathrm{C} 2} \quad$ Seasonal performance factor over 365 consecutive days for the system boundary $\mathrm{C} 2$ (cooling unit and circulation pumps/fans on source-side) 


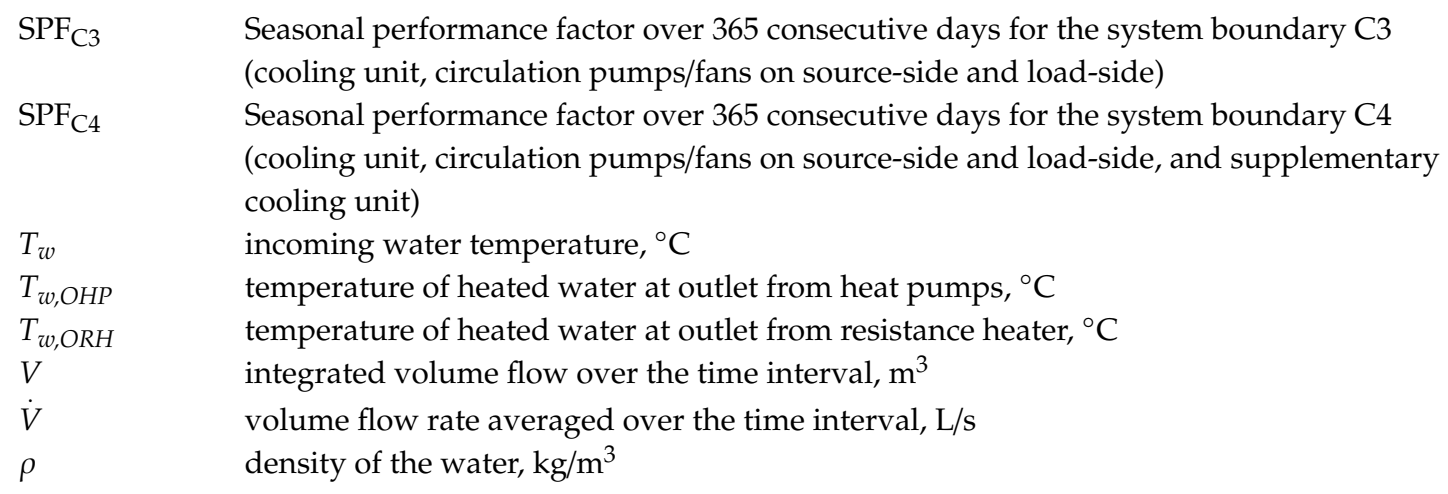

\section{Abbreviations}

$\begin{array}{ll}\text { COP } & \text { Coefficient of performance } \\ \text { DHW } & \text { Domestic hot water } \\ \text { EAHR } & \text { Exhaust air heat recovery } \\ \text { EFT } & \text { Entering fluid temperature } \\ \text { GSHP } & \text { Ground source heat pump } \\ \text { LPS } & \text { Legionella protection system } \\ \text { SPF } & \text { Seasonal performance factor }\end{array}$

\section{References}

1. Pérez-Lombard, L.; Ortiz, J.; Pout, C. A review on buildings energy consumption information. Energy Build. 2008, 40, 394-398. [CrossRef]

2. USEIA. How Much Energy Is Consumed in U.S. Residential and Commercial Buildings? Available online: https://www.eia.gov/tools/faqs/faq.php?id=86\&t=1 (accessed on 9 July 2018).

3. Swedish Energy Agency. Energy in Sweden Facts and Figures 2018; Swedish Energy Agency: Eskilstuna, Sweden, 2018.

4. Swedish Energy Agency. Energistatistik för Småhus, Flerbostadshus och Lokaler 2016. Summary of Energy Statistics for Dwellings and Non-Residential Premises for 2016. ES 2017:6; Swedish Energy Agency: Eskilstuna, Sweden, 2017.

5. De Wilde, P. The gap between predicted and measured energy performance of buildings: A framework for investigation. Autom. Constr. 2014, 41, 40-49. [CrossRef]

6. Imam, S.; Coley, D.A.; Walker, I. The building performance gap: Are modellers literate? Build. Serv. Eng. Res. Technol. 2017, 38, 351-375. [CrossRef]

7. Liang, J.; Qiu, Y.; Hu, M. Mind the energy performance gap: Evidence from green commercial buildings. Resour. Conserv. Recycl. 2019, 141, 364-377. [CrossRef]

8. Menezes, A.C.; Cripps, A.; Bouchlaghem, D.; Buswell, R. Predicted vs. actual energy performance of non-domestic buildings: Using post-occupancy evaluation data to reduce the performance gap. Appl. Energy 2012, 97, 355-364. [CrossRef]

9. Scofield, J.H. Do LEED-certified buildings save energy? Not really. Energy Build. 2009, 41, 1386-1390. [CrossRef]

10. Scofield, J.H. Efficacy of LEED-certification in reducing energy consumption and greenhouse gas emission for large New York City office buildings. Energy Build. 2013, 67, 517-524. [CrossRef]

11. Li, C.; Hong, T.; Yan, D. An insight into actual energy use and its drivers in high-performance buildings. Appl. Energy 2014, 131, 394-410. [CrossRef]

12. Kurkinen, E.-L.; Filipsson, P.; Elfborg, S.; Ruud, S. Skillnad Mellan Beräknad och Verklig Energianvändning-Energistyrning Under Byggprocessen—Slutrapport December 2014; SP Sveriges Tekniska Forskningsinstitut: Borås, Sweden, 2014.

13. Geng, Y.; Ji, W.; Wang, Z.; Lin, B.; Zhu, Y. A review of operating performance in green buildings: Energy use, indoor environmental quality and occupant satisfaction. Energy Build. 2019, 183, 500-514. [CrossRef] 
14. Mathew, P.A.; Dunn, L.N.; Sohn, M.D.; Mercado, A.; Custudio, C.; Walter, T. Big-data for building energy performance: Lessons from assembling a very large national database of building energy use. Appl. Energy 2015, 140, 85-93. [CrossRef]

15. Walter, T.; Sohn, M.D. A regression-based approach to estimating retrofit savings using the building performance database. Appl. Energy 2016, 179, 996-1005. [CrossRef]

16. Deng, H.; Fannon, D.; Eckelman, M.J. Predictive modeling for US commercial building energy use: A comparison of existing statistical and machine learning algorithms using CBECS microdata. Energy Build. 2018, 163, 34-43. [CrossRef]

17. Kontokosta, C.E.; Tull, C. A data-driven predictive model of city-scale energy use in buildings. Appl. Energy 2017, 197, 303-317. [CrossRef]

18. An, J.; Yan, D.; Hong, T. Clustering and statistical analyses of air-conditioning intensity and use patterns in residential buildings. Energy Build. 2018, 174, 214-227. [CrossRef]

19. USEPA. ENERGY STAR Certification for Your Building. Available online: https://www.energystar.gov/ buildings/facility-owners-and-managers/existing-buildings/earn-recognition/energy-star-certification (accessed on 9 July 2018).

20. EPRI. Long-Term Performance of Commercial Ground Source Heat Pumps; 1026421; EPRI: Palo Alto, CA, USA, 2012.

21. Gleeson, C.P.; Lowe, R. Meta-analysis of European heat pump field trial efficiencies. Energy Build. 2013, 66, 637-647. [CrossRef]

22. Nordman, R.; Kleefkens, O.; Riviere, P.; Nowak, T.; Zottl, A.; Arzano-Daurelle, C.; Lehmann, A.; Polyzou, O.; Karytsas, K.; Riederer, P.; et al. SEPEMO—Seasonal Performance Factor and Monitoring for Heat Pump Systems in the Building Sector; SP Technical Research Institute of Sweden: Borås, Sweden, 2012.

23. Miara, M.; Günther, D.; Kramer, T.; Oltersdorf, T.; Wapler, J. Heat Pump Efficiency-Analysis and Evaluation of Heat Pump Efficiency in Real-life Conditions. Abbreviated Version; Fraunhofer ISE: Freiburg, Germany, 2011.

24. Koenigsdorff, R. Oberflächennahe Geothermie für Gebäude Grundlagen und Anwendungen Zukunftsfähiger Heizung und Kühlung; Fraunhofer IRB: Stuttgart, Germany, 2011.

25. VDI. VDI 4650 Calculation of the Seasonal Coefficient of Performance of Heat Pumps. Electric Heat Pumps for Space Heating and Domestic Hot Water; VDI: Düsseldorf, Germany, 2016.

26. Karagiorgas, M.; Mendrinos, D.; Karytsas, C. Solar and geothermal heating and cooling of the European Centre for Public Law building in Greece. Renew. Energy 2003, 29, 461-470. [CrossRef]

27. Hwang, Y.; Lee, J.-K.; Jeong, Y.-M.; Koo, K.-M.; Lee, D.-H.; Kim, I.-K.; Jin, S.-W.; Kim, S.H. Cooling performance of a vertical ground-coupled heat pump system installed in a school building. Renew. Energy 2009, 34, 578-582. [CrossRef]

28. Kim, E.; Lee, J.; Jeong, Y.; Hwang, Y.; Lee, S.; Park, N. Performance evaluation under the actual operating condition of a vertical ground source heat pump system in a school building. Energy Build. 2012, 50, 1-6. [CrossRef]

29. Sebarchievici, C.; Dan, D.; Sarbu, I. Performance Assessment of a Ground-coupled Heat Pump for an Office Room Heating using Radiator or Radiant Floor Heating Systems. Procedia Eng. 2015, 118, 88-100. [CrossRef]

30. Zhou, S.; Cui, W.; Zhao, S.; Zhu, S. Operation analysis and performance prediction for a GSHP system compounded with domestic hot water (DHW) system. Energy Build. 2016, 119, 153-163. [CrossRef]

31. Zhou, Z.; Feng, L.; Zhang, S.; Wang, C.; Chen, G.; Du, T.; Li, Y.; Zuo, J. The operational performance of net zero energy building: A study in China. Appl. Energy 2016, 177, 716-728. [CrossRef]

32. Wemhoener, C. IEA HPP Annex 40-Switzerland Participation. Available online: https://www.annex40.net/ index.php?id=11581 (accessed on 23 July 2018).

33. Wemhoener, C.; Hässig, W.; Wyss, S.; Staubli, J. Heat pump application in nearly zero energy buildings. Sci. Technol. Built Environ. 2017, 23, 637-650. [CrossRef]

34. Lazzarin, R.; Noro, M. Lessons learned from long term monitoring of a multisource heat pump system. Energy Build. 2018, 174, 335-346. [CrossRef]

35. Busato, F.; Lazzarin, R.M.; Noro, M. Two years of recorded data for a multisource heat pump system: A performance analysis. Appl. Therm. Eng. 2013, 57, 39-47. [CrossRef]

36. Schmidt, T.; Müller-Steinhagen, H. The Central Solar Heating Plant with Aquifer Thermal Energy Store in Rostock-Results after four years of operation. In Proceedings of the EuroSun 2004-The 5th ISES Europe Solar Conference, Freiburg, Germany, 20-23 June 2004. 
37. Urchueguía, J.F.; Zacarés, M.; Corberán, J.M.; Montero, Á.; Martos, J.; Witte, H. Comparison between the energy performance of a ground coupled water to water heat pump system and an air to water heat pump system for heating and cooling in typical conditions of the European Mediterranean coast. Energy Convers. Manag. 2008, 49, 2917-2923. [CrossRef]

38. Montagud, C.; Corberán, J.M.; Montero, Á.; Urchueguía, J.F. Analysis of the energy performance of a ground source heat pump system after five years of operation. Energy Build. 2011, 43, 3618-3626. [CrossRef]

39. Hughes, D. Monitoring of Non-Domestic Renewable Heat Incentive Ground-Source and Water-Source Heat Pumps-Interim Report; Department of Energy \& Climate Change: London, UK, 2016.

40. Hughes, D. Monitoring of Non-Domestic Renewable Heat Incentive Ground-Source and Water-Source Heat Pumps_Case Studies; UK Department for Business, Energy \& Industrial Strategy; Department of Energy \& Climate Change: London, UK, 2018.

41. Hughes, D. Monitoring of Non-Domestic Renewable Heat Incentive Ground-Source \& Water-Source Heat Pumps-Final Report; UK Department for Business, Energy \& Industrial Strategy; Department of Energy \& Climate Change: London, UK, 2018.

42. Winiger, S.; Kalz, D.; Sonntag, M.; Vellei, M. Energy and Efficiency Analysis of Heat Pump Systems In Nonresidential Buildings by Means of Long-Term Measurements. In Proceedings of the 11th REHVA World Congress \& 8th International Conference on IAQVEC, CLIMA 2013, Prague, Czech Republic, 16-19 June 2013.

43. Mendrinos, D.; Karytsas, C. Monitoring results after 12 months of provision of heating and cooling at the 8 demonstration buildings of the European project "Ground-Med". In Proceedings of the European Geothermal Congress 2016, Strasbourg, France, 19-23 September 2016.

44. Zhai, X.Q.; Yang, Y. Experience on the application of a ground source heat pump system in an archives building. Energy Build. 2011, 43, 3263-3270. [CrossRef]

45. Vanhoudt, D.; Desmedt, J.; Van Bael, J.; Robeyn, N.; Hoes, H. An aquifer thermal storage system in a Belgian hospital: Long-term experimental evaluation of energy and cost savings. Energy Build. 2011, 43, 3657-3665. [CrossRef]

46. Michopoulos, A.; Zachariadis, T.; Kyriakis, N. Operation characteristics and experience of a ground source heat pump system with a vertical ground heat exchanger. Energy 2013, 51, 349-357. [CrossRef]

47. Wagner, A.; Lützkendorf, T.; Voss, K.; Spars, G.; Maas, A.; Herkel, S. Performance analysis of commercial buildings-Results and experiences from the German demonstration program Energy Optimized Building (EnOB). Energy Build. 2014, 68, 634-638. [CrossRef]

48. Mermoud, F.; Hollmuller, P.; Pampaloni, E.; Fraga, C.; Lachal, B. In situ monitoring results of an existing geothermal heat pump for the heating of a retrofitted office building in Geneva. In Proceedings of the 11th International Energy Agency Heat Pump Conference, Montreal, QC, Canada, 12-16 May 2014.

49. Southard, L.E.; Liu, X.; Spitler, J.D. Performance of HVAC Systems at ASHRAE HQ-Part 2. ASHRAE J. 2014, 56, 12-23.

50. Southard, L.E.; Liu, X.; Spitler, J.D. Performance of HVAC Systems at ASHRAE HQ-Part 1. ASHRAE J. 2014, 56, 14-24.

51. Spitler, J.D.; Southard, L.E.; Liu, X. Ground-source and air-source heat pump system performance at the ASHRAE headquarters building. In Proceedings of the 12th IEA Heat Pump Conference, Rotterdam, The Netherlands, 15-18 May 2017.

52. Fuentes, E.; Waddicor, D.A.; Salom, J. Improvements in the characterization of the efficiency degradation of water-to-water heat pumps under cyclic conditions. Appl. Energy 2016, 179, 778-789. [CrossRef]

53. Waddicor, D.A.; Fuentes, E.; Azar, M.; Salom, J. Partial load efficiency degradation of a water-to-water heat pump under fixed set-point control. Appl. Therm. Eng. 2016, 106, 275-285. [CrossRef]

54. Carvalho, A.D.; Mendrinos, D.; De Almeida, A.T. Ground source heat pump carbon emissions and primary energy reduction potential for heating in buildings in Europe-results of a case study in Portugal. Renew. Sustain. Energy Rev. 2015, 45, 755-768. [CrossRef]

55. Pardo, P.; Michal, H. D8.1: Synthesis of Systems Performance Evaluation in the Various Types of Buildings. Available online: http://groundmed.eu/deliverables/reports_and_papers/ (accessed on 27 December 2018).

56. Schibuola, L.; Scarpa, M. Ground source heat pumps in high humidity soils: An experimental analysis. Appl. Therm. Eng. 2016, 99, 80-91. [CrossRef]

57. Pater, S.; Ciesielczyk, W. Real and Theoretical Energy Efficiency of Vapour Compression Heat Pumps. Mechanics 2017, 5, 185-194. 
58. Garber-Slaght, R.; Peterson, R. Can Ground source heat pumps perform well in Alaska? In Proceedings of the IGSHPA Technical/Research Conference and Expo, Denver, CO, USA, 14-16 March 2017; pp. 62-70.

59. Liu, X.; Malhotra, M.; Im, P. Performance Analysis of Ground Source Heat Pump Demonstration Projects in the United States. In Proceedings of the 12th IEA Heat Pump Conference, Rotterdam, The Netherlands, 15-18 May 2017.

60. Naicker, S.S.; Rees, S.J. Performance analysis of a large geothermal heating and cooling system. Renew. Energy 2018, 122, 429-442. [CrossRef]

61. Gehlin, S.; Spitler, J.D.; Larsson, A.; Annsberg, Å. Measured performance of the University of Stockholm Studenthuset ground source heat pump system. In Proceedings of the EnerStock 2018, Adana, Turkey, 25-28 April 2018.

62. Xing, L.; Spitler, J.D.; Bandyopadhyay, A. Prediction of undisturbed ground temperature using analytical and numerical modeling. Part III: Experimental validation of a world-wide dataset. Sci. Technol. Built Environ. 2017, 23, 826-842. [CrossRef]

63. Bergqvist, B. Kartläggning av VVC-Förluster i Flerbostadshus-Mätningar i 12 Fastigheter—Slutrapport; BeBo: Stockholm, Sweden, 2015.

64. Bergqvist, B. VVC-Förluster i Kontor och Lokaler-Mätningar i 11 Byggnader-Slutrapport; BeBo: Stockholm, Sweden, 2016.

65. Taylor, J.R. An Introduction to Error Analysis—The Study of Uncertainties in Physical Measurements, 2nd ed.; University Science Books: Sausalito, CA, USA, 1997.

66. IEA. HPC Annex 52-Long Term Performance Measurement of GSHP Systems Serving Commercial, Institutional and Multi-Family Buildings. Available online: https:/heatpumpingtechnologies.org/annex52/ (accessed on 1 May 2019).

(C) 2019 by the authors. Licensee MDPI, Basel, Switzerland. This article is an open access article distributed under the terms and conditions of the Creative Commons Attribution (CC BY) license (http://creativecommons.org/licenses/by/4.0/). 